Federal Reserve Bank of Minneapolis Research Department

\title{
Search-Based Models of Money and Finance: An Integrated Approach*
}

\author{
Alberto Trejos and Randall Wright \\ Working Paper 709
}

March 2014

\begin{abstract}
Many applications of search theory in monetary economics use the Shi-Trejos-Wright model, hereafter STW, while applications in finance use Duffie-Gârleanu-Pederson, hereafter DGP. These approaches have much in common, and both claim to be about liquidity, but the models also differ in a fundamental way: in STW agents use assets as payment instruments when trading goods; in DGP there are no gains from exchanging goods, but agents trade because they value assets differently with goods serving as payment instruments. We develop a framework nesting the two. This clarifies the connection between the literatures, and generates new insights and applications. Even in the special cases of the baseline STW and DGP models, we provide propositions generalizing and strengthening what is currently known, and rederiving some existing results using more tractable arguments.
\end{abstract}

Keywords: Search; Bargaining; Money; Finance

JEL classification: E40, E44

*Trejos: INCAE and University of Michigan. Wright: University of Wisconsin-Madison, Federal Reserve Bank of Minneapolis, Federal Reserve Bank of Chicago, and NBER. Corresponding Author: R. Wright, email: rwright@bus.wisc.edu., phone: 608-770-7919, fax: 608-262-3656. We thank many friends and colleagues for their input, including Cyril Monnet, Chao Gu, Guillaume Rocheteau, Yu Zhu, Ricardo Lagos, Karl Shell, and especially Huberto Ennis, Lasse Pederson, and Ana Babus, who served as formal discussants. We also thank workshop organizers and participants at FRB Chicago, Arhus, Wisconsin, Newcastle, INCAE, Cornell and Carnegie. Wright thanks the NSF and the Ray Zemon Chair in Liquid Assets at the Wisconsin School of Business for research support. The views expressed herein are those of the authors and not necessarily those of the Federal Reserve Bank of Minneapolis or the Federal Reserve System. 


\section{Introduction}

One of our jobs as scholars is to explore connections between disparate theories, or between models that seem related yet different. In search-and-bargaining theory, many applications in monetary economics build on Shi (1995) or Trejos and Wright (1995), hereafter STW, while applications in finance build on Duffie, Gârleanu and Pederson (2005), hereafter DGP. ${ }^{1}$ A search-and-bargaining approach is natural in monetary theory, as it provides a convenient way to study bilateral trade when barter and credit are difficult. In finance, it is not only natural, but accurate: "Many assets, such as mortgage-backed securities, corporate bonds, government bonds, US federal funds, emerging-market debt, bank loans, swaps and many other derivatives, private equity, and real estate, are traded in over-the-counter (OTC) markets. Traders in these markets search for counterparties, incurring opportunity or other costs. When counterparties meet, their bilateral relationship is strategic; prices are set through a bargaining process that reflects each investor's alternatives to immediate trade" (Duffie et al. 2007, emphasis added).

Once one goes beyond superficial issues of notation and interpretation, it is apparent that the STW and DGP models are closely related, and both claim to be about liquidity. As we show, neither is a special case of the other, but there is still a connection that is worth making precise. We provide an integrated framework nesting STW and DGP as special cases. This is useful because people pursuing applications in finance with these models ought know there is similar work in monetary economics,

\footnotetext{
${ }^{1}$ Here is a literature review, that one can skip for now if so inclinded. Both STW and DGP use what New Monetarists (see Williamson and Wright 2010 or Nosal and Rocheteau 2011 for surveys) call second-generation models, where assets are indivisible and agents can hold at most 1. Firstgeneration models had indivisible goods, too. For third-generation models, with divisible assets and goods, in STW we have Shi (1997), Green and Zhou (1998), Lagos and Wright (2005), Rocheteau and Wright (2005), and Molico (2006); in DGP we have Lagos and Rocheteau (2009), Lagos et al. (2011), Babus and Kondor (2012), and Monnet and Narajabad (2012). Despite these extensions, indivisible-asset models are still useful, and more tractable. See, e.g., Wallace (2000,2010), Trejos and Wright (1993,2001), He et al. (2005), Wallace and Nosal (2007), Wallace and Zhu (2007a,b), Ales et al. (2010), and Choi (2012), who use STW, and Duffie et al. (2007), Weill (2007,2008), Weill and Vayanos (2008), Pagnotta and Philippon (2011), and Chiu and Koeppl (2012) who use DGP.
} 
and vice versa. Also, integrating the approaches allows insights from one field to help understand substantive issues in the other. Since people in finance, e.g., are interested in bubbles, excess volatility and related phenomena, monetary theory has a lot to contribute in terms of methods and results. Since many assets are increasingly used to facilitate transactions, e.g., T-bills serving as collateral or housing wealth securing home-equity loans, monetary economists could benefit from knowing more finance. One of our goals is to bridge some gaps between the two fields.

It has been said that "some papers are about issues, and other papers are about papers." It is natural to think of this as one of the latter type - the motivation is integrating two popular literatures in monetary and financial economics. It is no surprise that people familiar with these literatures will see some things that look familiar, but there are also several original contributions: even in the special cases of the baseline STW and DGP models, we provide propositions that generalize and strengthen what is currently known, and we rederive some existing results using more tractable arguments. To better understand what is novel and what constitutes a review, here are ten things that are new relative to existing work.

1. As regards the STW model, we allow assets to bear a flow return $\delta$. Most presentations of the model have $\delta=0$ (fiat money); a few allow $\delta>0$ (dividends); even fewer consider $\delta<0$ (storage cost). We characterize steady states and perfect-foresight dynamics for all $\delta$ (Proposition 1). In particular, we prove that $\delta<0$ implies the asset can be valued and circulate as a medium of exchange iff $\delta$ is not too big, and $\delta>0$ implies it is always valued but circulates iff $\delta$ is not too big. Although parts of these results can be found elsewhere, there is no such comprehensive characterization.

2. While there are papers showing the existence of sunspot equilibria in STW (e.g., Shi 1995 or Ennis 2001,2004), we show this using different methods, and show such equilibria exist for a rather larger set of parameters (Proposition 2). As 
excess volatility is one reason to be interested in STW, it is useful to know more about sunspot equilibria in the model.

3. Different from previous analyses of STW, we use Kalai bargaining, instead of generalized Nash. The results are similar, but also differ in interesting ways. Kalai allows us to derive stronger results, and to derive them more easily. Moreover, for a different-but-related class of models following Lagos and Wright (2005), it has been argued by Aruoba et al. (2007) that Kalai bargaining has advantages over Nash in addition to tractability: it guarantees value functions are concave; it provides no incentive to hide assets; it gives cleaner predictions; and so on. Hence, in those models the standard approach is now Kalai bargaining, and we think it should be that way in STW, too. This is the first analysis of STW with Kalai bargaining.

4. As regards the baseline DGP model, which has $u(q)=q$, we allow concave utility over transfers going to the agent giving up the asset. Many results in the baseline model go through, but others change - e.g., there is generally no longer a unique steady state or a unique equilibrium (Proposition 10). This is new.

5. Different from the baseline DGP model, nonlinear $u(q)$ implies trading patterns can be much more interesting: with $u(q)=q$, trade only occurs when an agent with an asset and a low valuation for it meets one with a high valuation without the asset; here, agents with the same valuation may trade, and sometimes even agents with high valuations trade assets to those with low valuations. This is endogenous, and depends on parameter values (Proposition 9).

6. In DGP, even with $u(q)=q$, while it is known there is a unique steady state, we show much more: when $u(q)=q$ there is a unique equilibrium. We do this by verifying explicitly saddle-path stability for steady state (Proposition 4). This 
may be merely a matter of calculation, but it is important because it implies not only that the baseline model cannot have multiple steady states, it implies it cannot have any endogenous multiplicity.

7. Although it is known that the baseline DGP model has a unique steady state for a given trading pattern, we calculate the closed-form solution explicitly. While this is again merely a matter of calculation, it can be useful in applications to have closed-form solutions. Similarly, in terms of calculations, we provide explicit comparative-static results for DGP (Proposition 5) and STW (Proposition 3) which are cleaner than existing results.

8. In models with indivisible assets, since trading opportunities are not convex, there can be a role for lotteries in facilitating exchange. While there are previous analyses of STW that allow lotteries (e.g., Berentsen et al. 2002), they typically restrict attention to $\delta=0$. We analyze the model for general $\delta$ and, in particular, show that for $\delta<0$, but not for $\delta>0$, there can be multiple steady states when lotteries are allowed (Figure 5).

9. While DGP models have been studied with dealers that intermediate between asset buyers and sellers (as we review in Proposition 7), we consider different types of intermediation, including banks that in addition to trading the exogenous stock of assets can issue their own assets. This generates new results, relative to previous analyses of this model, such as inside liquidity getting crowded out by changes in outside liquidity (Proposition 8).

10. We provide a way to embed STW and DGP models into a simple general equilibrium setting (Section 5), where agents trade different consumption goods and labor. This allows us to study a much broader set of issues - e.g., the effect of activity in asset markets on labor markets - compared to previous analyses of these models. 
The rest of the paper is organized as follows. Section 2 lays out a general description of an OTC market. Section 3 provides results for a special case where the framework collapses to STW. Section 4 provides results when it collapses to DGP. Section 5 shows how to embed simple OTC markets into general equilibrium. Section 6 analyzes the integrated model. Section 7 concludes.

\section{The Environment}

Time is continuous (but see Section 5) and the horizon infinite. There is a $[0,1]$ continuum of agents that discount at rate $r>0$. They meet, and potentially trade, bilaterally and anonymously according to a Poisson process with arrival rate $\alpha$. There are two tradable objects: a nonstorable consumption good $q$ and a storable asset $m$. As in STW and DGP, the asset is indivisible, and agents can hold at most 1 unit. Thus, if $M \in(0,1)$ is the fixed supply of the asset, $M$ is also the measure of agents holding $m=1$ while $1-M$ is the measure holding $m=0$. One can think of the asset is a standard Lucas (1978) "tree" giving off a constant dividend stream, normalized to 1 , in terms of "fruit," but agents may differ over time in their valuation of this dividend. Specifically, each individual has a state $\delta \in\left\{\delta_{L}, \delta_{H}\right\}$, which means that at that moment he gets either utility $\delta_{L}$ or $\delta_{H} \geq \delta_{L}$ from the dividend.

The state of an individual changes over time according to a Poisson process, independent of the matching process, with $\lambda_{H}$ denoting the rate of switching from $\delta_{H}$ to $\delta_{L}$ and $\lambda_{L}$ denoting the rate of switching back. ${ }^{2}$ In DGP, there are potential gains from trade when an agent in state $\delta_{H}$ with $m=0$ meets someone in state $\delta_{L}$ with $m=1$. Some papers using the DGP model refer to a switch from $\delta_{H}$ to $\delta_{L}$ as a liquidity shock, since it makes agents want to divest themselves of assets. In contrast, in STW, $\delta_{L}=\delta_{H}=\delta$, where the original papers focus on $\delta=0$, which

\footnotetext{
${ }^{2}$ One can imagine $\delta_{H}>\delta_{L}$ is due to individual asset-holding costs. Or, Duffie et al. (2007) say it can be due to: "(i) low liquidity, that is, a need for cash, (ii) high financing or financial-distress costs, (iii) adverse correlation of asset returns with endowments ... (iv) a relative tax disadvantage ... (v) a relatively low personal use for ... certain durable consumption goods such as homes."
} 
means the asset is fiat money - i.e., an object that is "intrinsically useless" (Wallace 1980). Hence there are no gains from exchanging assets in STW; instead, gains from trade arise due to the production and consumption of $q$.

There are many ways to specify specialization. Following Aiyagari and Wallace (1991), we assume there are $K$ varieties of the good and equal measures of $K$ types of agents, where type $k$ consumes variety $k$ and produces $k+1$ modulo $K$. One meets someone who produces something one desires at rate $\sigma=\alpha / K$. As long as $K>2$ there can be no barter, and credit is impossible since agents are anonymous (Kocherlakota 1998). Therefore, when an agent wants another agent to produce, if the former has an asset and the latter does not, they can potentially swap $m$ for $q$. Economists using STW interpret assets in the model as valued for their liquidity, in a different sense than the one used in DGP: for the latter, the sale of an asset is motivated by a need for liquidity; for the former, the asset is liquidity.

Let $u(q)$ be the utility from consuming $q$ units of one's consumption good, and $c(q)$ the disutility of producing, with $u(0)=c(0)=0, u^{\prime}(q)>0$ and $c^{\prime}(q)>0$. In STW, $u^{\prime \prime}(q) \leq 0$ and $c^{\prime \prime}(q) \geq 0$, with at least one strict, in which case we define $\bar{q}>0$ by $u(\bar{q})=c(\bar{q})$ and $q^{*} \in(0, \bar{q})$ by $u^{\prime}\left(q^{*}\right)=c^{\prime}\left(q^{*}\right)$. In DGP, $u(q)=c(q)=q$, so there are no gains from trade in $q$, and as we said, it is simply a means of payment when trading assets. This completes our description of the basic environment. Since we want to highlight symmetries between the models, for now, we ignore some details, such as the ability to barter or use credit in some meetings in STW, or the fact that in some versions of DGP agents can trade with intermediaries (we come back to this in Section 4, where intermediation is discussed explicitly).

To discuss outcomes, first, let $n_{m}^{i}$ be the measure of agents holding $m \in\{0,1\}$ with $\delta=\delta_{i} \in\left\{\delta_{H}, \delta_{L}\right\}$. Let $\phi_{b s}$ be the probability of trade when an agent with $\delta_{b} \in\left\{\delta_{H}, \delta_{L}\right\}$ and $m=1$ meets an agent with $\delta_{s} \in\left\{\delta_{H}, \delta_{L}\right\}$ and $m=0$, where subscripts $b$ and $s$ indicate the potential buyer and seller of $q$. Of course, the seller 
of $q$ is the buyer of $m$, and vice-versa; unless explicitly stated to the contrary, by the buyer we mean the buyer of $q$. Labelling aside, the law of motion for $n_{1}^{L}$ is

$$
\dot{n}_{1}^{L}=\lambda_{H} n_{1}^{H}-\lambda_{L} n_{1}^{L}-\sigma n_{1}^{L} n_{0}^{H} \phi_{L H}+\sigma n_{0}^{L} n_{1}^{H} \phi_{H L}
$$

The first term on the RHS is the flow in due to agents with $m=1$ switching from $\delta_{H}$ to $\delta_{L}$. The second is the flow out due to agents with $m=1$ switching the other way. The third term is the flow out due to agents with $m=1$ and $\delta_{L}$ trading away assets to those with $m=0$ and $\delta_{H}$. The final term is the flow in due to agents with $m=0$ and $\delta_{L}$ acquiring assets from those with $m=1$ and $\delta_{H} \cdot{ }^{3}$

The rest of the dynamics are described by

$$
\begin{aligned}
\dot{n}_{0}^{H} & =\lambda_{L} n_{0}^{L}-\lambda_{H} n_{0}^{H}-\sigma n_{0}^{H} n_{1}^{L} \phi_{L H}+\sigma n_{1}^{H} n_{0}^{L} \phi_{H L} \\
1-M & =n_{0}^{H}+n_{0}^{L} \\
M & =n_{1}^{H}+n_{1}^{L} .
\end{aligned}
$$

Since (3)-(4) are identities, we can reduce this system to two equations in $\left(n_{1}^{L}, n_{0}^{H}\right)$, which is natural in DGP, where in equilibrium $\phi_{L H}=1$ and $\phi_{L L}=\phi_{H H}=\phi_{H H}=0$. It is natural in DGP because, while the total asset supply $M$ is fixed, $n_{1}^{L}$ represents the effective supply on the market: the measure of agents with an asset trying to trade it away. Similarly, $n_{0}^{H}$ represents the effective demand: the measure of agents with no asset trying to acquire one. In the general case, however, potentially anyone may trade for an asset (even those with $\delta_{L}$ ) or trade one away (even those with $\delta_{H}$ ).

As in Proposition 1 of Duffie et al.(2005), or as a special case of the proof of Lemma 2 below, we have:

Lemma 1 The system (1)-(4) has a unique, globally stable, steady state.

\footnotetext{
${ }^{3}$ This assumes random search, as in most of the literature, but one can also use directed search. Given our specialization pattern, e.g., we can assume type $k$ always directs his search to type $k-1$, who produces the good he wants. Still there is an element of randomness, since sometimes the producer of his good already has $m=1$, so they cannot trade, but one could change that and make search even more directed. See Coles (1999), Corbae et al. (2003), Matsu and Shimizu (2005) or Julien et al. (2008) for related models with alternative matching processes.
} 
Let $V_{m}^{i}$ be the payoff or value function for an agent holding $m \in\{0,1\}$ with $\delta=\delta_{i} \in\left\{\delta_{L}, \delta_{H}\right\}$. For $\delta=\delta_{H}$ and $m=1$, the flow Bellman equation is

$$
\begin{aligned}
r V_{1}^{H}= & \sigma n_{0}^{H} \phi_{H H}\left[u\left(q_{H H}\right)+V_{0}^{H}-V_{1}^{H}\right]+\sigma n_{0}^{L} \phi_{H L}\left[u\left(q_{H L}\right)+V_{0}^{H}-V_{1}^{H}\right] \\
& +\delta_{H}+\lambda_{H}\left(V_{1}^{L}-V_{1}^{H}\right)+\dot{V}_{1}^{H}
\end{aligned}
$$

The first term on the RHS is the rate of meeting someone who has a high valuation for the dividend but no asset $\sigma n_{0}^{H}$, times the probability of trade $\phi_{H H}$, times the surplus $u\left(q_{H H}\right)+V_{0}^{H}-V_{1}^{H}$, where $q_{b s}$ is the amount a buyer in state $b \in\{H, L\}$ gets from a seller in state $s \in\{H, L\}$ if they trade. The second term is the expected gain from trading with someone who has a low valuation. The other terms are the utility from dividends, the expected capital gain from switching states, and a pure time change $\dot{V}_{1}^{H}$. Similarly,

$$
\begin{aligned}
r V_{1}^{L}= & \sigma n_{0}^{H} \phi_{L H}\left[u\left(q_{L H}\right)+V_{0}^{L}-V_{1}^{L}\right]+\sigma n_{0}^{L} \phi_{L L}\left[u\left(q_{L L}\right)+V_{0}^{L}-V_{1}^{L}\right] \\
& +\delta_{L}+\lambda_{L}\left(V_{1}^{H}-V_{1}^{L}\right)+\dot{V}_{1}^{L} \\
r V_{0}^{H}= & \sigma n_{1}^{H} \phi_{H H}\left[V_{1}^{H}-V_{0}^{H}-c\left(q_{H H}\right)\right]+\sigma n_{1}^{L} \phi_{L H}\left[V_{1}^{H}-V_{0}^{H}-c\left(q_{L H}\right)\right] \\
& +\lambda_{H}\left(V_{0}^{L}-V_{0}^{H}\right)+\dot{V}_{0}^{H} \\
r V_{0}^{L}= & \sigma n_{1}^{H} \phi_{H L}\left[V_{1}^{L}-V_{0}^{L}-c\left(q_{H L}\right)\right]+\sigma n_{1}^{L} \phi_{L L}\left[V_{1}^{L}-V_{0}^{L}-c\left(q_{L L}\right)\right] \\
& +\lambda_{L}\left(V_{0}^{H}-V_{0}^{L}\right)+\dot{V}_{0}^{L} .
\end{aligned}
$$

If we let $\Delta^{i}=V_{1}^{i}-V_{0}^{i}$ for $i \in\{L, H\}$, equilibrium trading strategies satisfy

$$
\phi_{b s}= \begin{cases}1 & \text { if } u\left(q_{b s}\right)>\Delta^{b} \text { and } c\left(q_{b s}\right) \leq \Delta^{s} \\ 1 & \text { if } u\left(q_{b s}\right) \geq \Delta^{b} \text { and } c\left(q_{b s}\right)<\Delta^{s} \\ 0 & \text { if } u\left(q_{b s}\right)<\Delta^{b} \text { and } c\left(q_{b s}\right) \geq \Delta^{s} \\ 0 & \text { if } u\left(q_{b s}\right) \leq \Delta^{b} \text { and } c\left(q_{b s}\right)>\Delta^{s} \\ \Phi & \text { if } u\left(q_{b s}\right)=\Delta^{b} \text { and } c\left(q_{b s}\right)=\Delta^{s}\end{cases}
$$

where $\Phi$ is our notation for a generic mixed strategy. We also need to determine $q_{b s}$. While various mechanisms can be used, this paper adopts Kalai's (1977) proportional bargaining solution. Kalai bargaining in this context says that when a buyer with 
$\delta_{b}$ gives $m$ to a seller with $\delta_{s}$ for $q$, the former gets a share $\theta$ of the total surplus: $u\left(q_{b s}\right)-\Delta^{b}=\theta\left[u\left(q_{b s}\right)-\Delta^{b}-c\left(q_{b s}\right)+\Delta^{s}\right]$. This simplifies to

$$
(1-\theta) \Delta^{b}+\theta \Delta^{s}=(1-\theta) u\left(q_{b s}\right)+\theta c\left(q_{b s}\right) \equiv g\left(q_{b s}\right)
$$

where the function $g(\cdot)$ plays a big role in what follows. ${ }^{4}$

Define the vectors $\mathbf{n}=\left(n_{m}^{i}\right), \mathbf{V}=\left(V_{m}^{i}\right), \boldsymbol{\phi}=\left(\phi_{b s}\right)$ and $\mathbf{q}=\left(q_{b s}\right)$. Then we have:

Definition 1 Given an initial $\mathbf{n}_{0}$, equilibrium is a list $\{\mathbf{n}, \mathbf{V}, \boldsymbol{\phi}, \mathbf{q}\}$ of nonnegative and bounded functions of time satisfying: the law of motion (1)-(4) for $\mathbf{n}$; the Bellman equations (5)-(8) for $\mathbf{V}$; the trading conditions (9) for $\boldsymbol{\phi}$; and the bargaining solution (10) for q. A stationary equilibrium, or steady state, is one that is constant wrt time.

Appendix A presents an extended model with $\delta_{i} \in\left\{\delta_{1}, \delta_{2}, \ldots \delta_{I}\right\}$ for any integer $I$, and provides an algorithm like the one in Kiyotaki and Wright (1989) to solve for the set of stationary pure-strategy equilibria. For now, we use $I=1$ as in STW or $N=2$ as in DGP to discuss substantive applications in money and finance.

\section{Money}

Suppose $\delta_{L}=\delta_{H}=\delta$, as in STW, except that we use Kalai bargaining, which makes the model more tractable, and we allow $\delta \neq 0$, which makes it more interesting. Indeed, we not only allow $\delta>0$, as in DGP, we also consider $\delta<0$, in which case $\delta$ can be interpreted as a storage cost. When $\delta_{i}=\delta$, we ignore superscripts and write

\footnotetext{
${ }^{4}$ Aruoba et al. (2007) discuss the advantages of Kalai bargaining in a class of related models. With $u(q)=c(q)=q$, as in DGP, Kalai is equivalent to generalized Nash bargaining. The original STW models use a bargaining game that is equivalent to symmetric Nash; Rupert et al. (2001) extend this to generalized Nash. Other solution concepts used in these models include pure mechanism design (Wallace and Zhu 2007a,b), price posting (Curtis and Wright 2004; Julien et al. 2008), and, in versions with some multilateral meetings, auctions (Julien et al.2008). For comparison, the generalized Nash bargaining solution in this model implies

$$
(1-\theta) c^{\prime}\left(q_{b s}\right) \Delta^{b}+\theta u^{\prime}\left(q_{b s}\right) \Delta^{s}=(1-\theta) c^{\prime}\left(q_{b s}\right) u\left(q_{b s}\right)+\theta u^{\prime}\left(q_{b s}\right) c\left(q_{b s}\right)
$$

Unless $u(q)=c(q)=q$, or $\theta=1$, this differs from (10) for $q \neq q^{*}$. While the qualitative results are similar, Kalai bargaining considerably reduces the algebra compared to Nash.
} 
$V_{m}^{i}=V_{m}$ and $n_{m}^{i}=n_{m}$. The distribution is summarized by $n_{1}=M$ and $n_{0}=1-M$, which are constant over time, even if other variables are not. Moreover, $\phi_{b s}=\phi$, and $q_{b s}=q \forall(b, s)$, so there is a single $q$ giving the price of the asset in terms of the good. Then bargaining implies $g(q)=\Delta$, and

$$
\begin{aligned}
& r V_{1}=\sigma(1-M) \phi\left[u(q)+V_{0}-V_{1}\right]+\delta+\dot{V}_{1} \\
& r V_{0}=\sigma M \phi\left[-c(q)+V_{1}-V_{0}\right]+\dot{V}_{0} .
\end{aligned}
$$

To solve the model, assume that trade occurs (i.e., $\phi=1$ ), and subtract the Bellman equations to get $\Delta$ as a function of $\dot{\Delta}$. Then, using the bargaining solution to eliminate $\Delta=g(q)$ and $\dot{\Delta}=g^{\prime}(q) \dot{q}$, we arrive at the differential equation

$$
g^{\prime}(q) \dot{q}=[r+\sigma M-(r+\sigma) \theta] u(q)-[\sigma M-(r+\sigma) \theta] c(q)-\delta \equiv e(q)
$$

where $e(q)$ also plays a big role in what follows. Equilibrium is given by a nonnegative and bounded solution to (11). To reduce notation, write $e(q)=A_{u} u(q)-A_{c} c(q)-\delta$, where $A_{u}=r+\sigma M-(r+\sigma) \theta$ and $A_{c}=\sigma M-(r+\sigma) \theta$. Notice

$$
A_{u}>0 \text { iff } \theta<\theta_{u} \equiv \frac{r+\sigma M}{r+\sigma} \text { and } A_{c}>0 \text { iff } \theta<\theta_{c} \equiv \frac{\sigma M}{r+\sigma}
$$

where $\theta_{c} \in(0, M)$ and $\theta_{u} \in(M, 1)$. This aids us in signing the derivatives

$$
e^{\prime}(q)=A_{u} u^{\prime}(q)-A_{c} c^{\prime}(q) \text { and } e^{\prime \prime}(q)=A_{u} u^{\prime \prime}(q)-A_{c} c^{\prime \prime}(q) .
$$

Also notice $e(0)=-\delta$ and $e(\bar{q})=\bar{\delta}-\delta$, where $u(\bar{q})=c(\bar{q})$ and $\bar{\delta}=r u(\bar{q})$.

Exchange requires $q \in[0, \bar{q}]$, since otherwise $u(q)<c(q)$ and trade is not mutually advantageous. A steady state is a $q^{e} \in[0, \bar{q}]$ solving $e\left(q^{e}\right)=0$. To analyze steady states, we break the analysis into three cases depending on $\theta$, as well as subcases depending on $\delta$.

Case 1: $\theta \in\left(\theta_{u}, 1\right]$. From (12)-(13), $A_{u}, A_{c}<0$ and $e(q)$ is convex. As Figure 1 shows, for $\delta>\bar{\delta}$ there is no solution to $e(q)=0$ in $(0, \bar{q})$, and hence there is no steady state with trade. Intuitively, when $\delta>\bar{\delta}$ the asset is so valuable that an agent will 

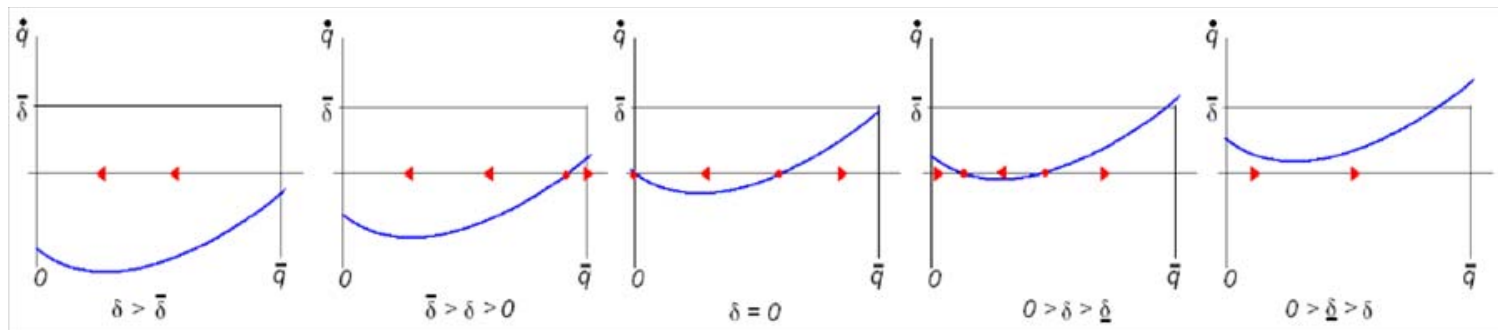

Figure 1: Existence of equilibria with trade, Case 1: $\theta>\theta_{u}$

only give it up for $q>\bar{q}$, but then agents with $m=0$ do not want it. For $\delta \in(0, \bar{\delta})$, there is a unique $q^{e} \in(0, \bar{q})$ solving $e\left(q^{e}\right)=0$, and hence a unique steady state with trade. For fiat currency, $\delta=0$, there is a nonmonetary steady state $q=0$ and a monetary steady state $q^{e} \in(0, \bar{q})$. For $\delta \in(\underline{\delta}, 0)$, where $\underline{\delta}<0$ is the value of $\delta$ that makes $\min _{q} e(q)=e(\underline{q})=0$, there are two steady states with trade, $q_{H}^{e} \in(0, \bar{q})$ and $q_{L}^{e} \in\left(0, q_{H}^{e}\right)$. And for $\delta<\underline{\delta}$ there is again no steady state with trade, but since $\delta<0$, now agents dispose of their assets.
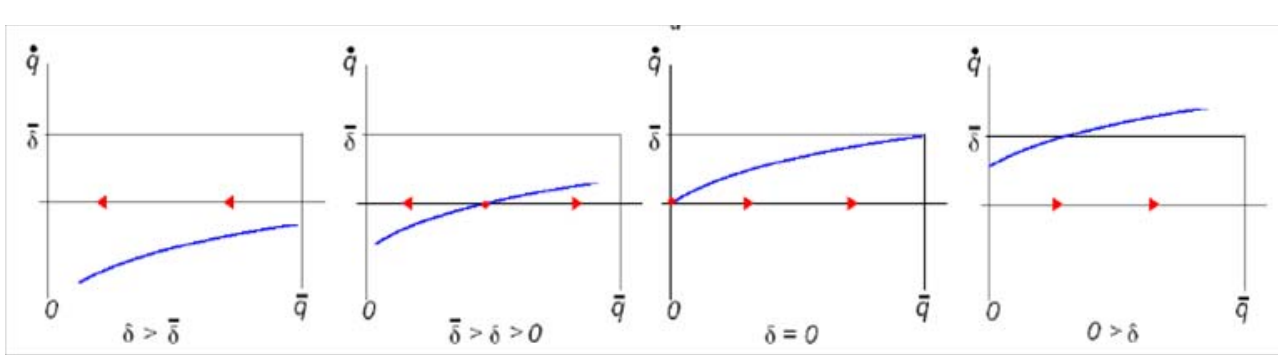

Figure 2: Existence of equilibria with trade, Case 2: $\theta_{u}>\theta>\theta_{c}$

Case 2: $\theta \in\left(\theta_{c}, \theta_{u}\right]$. Now (12)-(13) imply $A_{u}<0<A_{c}$ and $e^{\prime}(q)>0$. As Figure 2 shows: for $\delta>\bar{\delta}$ there is no steady state with trade and agents hoard assets; for $\delta \in(0, \bar{\delta})$ there is a unique steady state with trade $q^{e} \in(0, \bar{q})$; for $\delta=0$ nonmonetary steady state exists uniquely; and for $\delta<0$ agents dispose of assets.

Case 3: $\theta \in\left[0, \theta_{c}\right]$. Now $0<A_{c}, A_{u}$ and $e(q)$ is concave. Let $\hat{\delta}>\bar{\delta}$ be the $\delta$ that makes $\max _{q} e(q)=e(\hat{q})=0$. Given $\delta=\hat{\delta}$ there are two possibilities: Case 3a where $\hat{q}<\bar{q}$, and Case $3 \mathrm{~b}$ where $\hat{q} \geq \bar{q}$. Case $3 \mathrm{a}$ is shown in Figure 3. For $\delta>\hat{\delta}$ there is no 


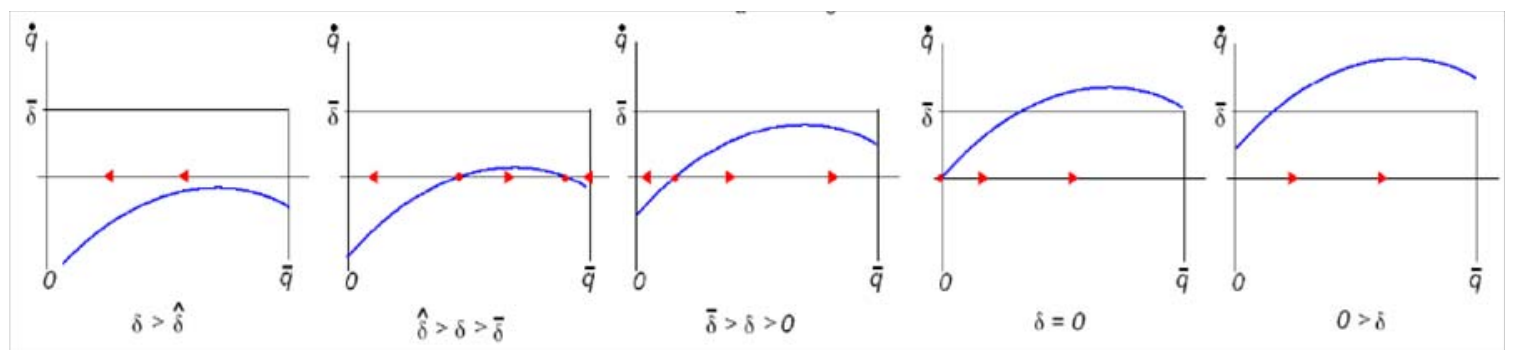

Figure 3: Existence of equilibria with trade, Case 3: $\theta<\theta_{c}$

steady state with trade; for $\delta \in(\bar{\delta}, \hat{\delta})$ there are two, $q_{H}^{e} \in(0, \bar{q})$ and $q_{L}^{e} \in\left(0, q_{H}^{e}\right)$; for $\delta \in(0, \bar{\delta})$ there is a unique $q^{e} \in(0, \bar{q})$; for $\delta=0$ the unique steady state is $q=0$; and for $\delta<0$ agents dispose of assets. ${ }^{5}$

To highlight a few economic results, first, $\delta$ can be so big that assets stop circulating - they get hoarded à la Gresham's Law. ${ }^{6}$ Second, if $\delta<0$ assets can be valued, but if $\delta<0$ is too big they will be abandoned. When $\delta=0$ the asset cannot circulate if $\theta$ is low, but if $\delta>0$ it may circulate even if $\theta=0$. Also, multiple steady states can arise for $\delta<0$ and for $\delta>0$. Multiplicity arises because the value of a liquid asset is at least partly a self-fulfilling property: if you think others will give a low $q_{L}$ for the asset then you will only give a low $q_{L}$ to get it; but if you think they will give a high $q_{H}$ then you will give more to get it. The pure version of this is fiat currency, since $\delta=0$ implies that, at least if $\theta>\theta_{u}$, there is always a monetary steady state with $q>0$ plus a nonmonetary one with $q=0$. But the logic applies to assets, too.

Next, consider perfect-foresight dynamics, which can be easily understood from Figures 1-3, since $\dot{q}=e(q) / g^{\prime}(q) \approx e(q)$, where $A \approx B$ means $A$ and $B$ have the same sign.

Case 1: $\theta \in\left(\theta_{u}, 1\right]$. In Figure 1, if $\delta>\bar{\delta}$ then $\dot{q}<0 \forall q \in[0, \bar{q}]$, so any path satisfying (11) leads to $q<0$, and the only equilibrium has no trade. If $\delta<\underline{\delta}<0$ then $\dot{q}>0$

\footnotetext{
${ }^{5}$ Case $3 \mathrm{~b}$ is only slightly different: in the second panel of Figure 3 there is a unique steady state, because $\hat{q}>\bar{q}$ implies the higher root of $e(q)=0$ exceeds $\bar{q}$. Note that Case 3a obtains for small $r$ and Case $3 \mathrm{~b}$ for big $r$.

${ }^{6}$ This might be more clear if we added a second tradable asset - which is not hard (see, e.g., Burdett et al. 2001 or Velde et al.1999), but we think there is enough going on already.
} 
$\forall q \in[0, \bar{q}]$, and the only equilibrium has agents disposing of $m$. For $\delta \in(0, \bar{\delta})$, any solution to (11) other than steady state $q^{e}$ leads either to $q<0$ or $q>\bar{q}$, so $q^{e}$ is the unique equilibrium. For $\delta=0$ there are two steady states, $q=0$ and $q^{e}>0$. Starting at any $q_{0}>q^{e}$ leads to $q>\bar{q}$, inconsistent with equilibrium. But starting at $q_{0} \in\left(0, q^{e}\right)$ constitutes an equilibrium where $q \rightarrow 0$. For $\delta \in(\underline{\delta}, 0)$, in addition to steady states $q_{H}^{e} \in(0, \bar{q})$ and $q_{L}^{e} \in\left(0, q_{H}^{e}\right)$, there is a continuum of equilibria, but now $q_{0} \in\left(0, q_{L}^{e}\right)$ implies $q_{t} \rightarrow q_{L}^{e}$ from below and $q_{0} \in\left(q_{L}^{e}, q_{H}^{e}\right)$ implies $q_{t} \rightarrow q_{L}^{e}$ from above.

Case 2: $\theta \in\left(\theta_{c}, \theta_{u}\right]$. In Figure 2, the only interesting situation (the only one with trade) is $\delta \in(0, \bar{\delta})$, where the steady state is the unique equilibrium.

Case 3: $\theta \in\left[0, \theta_{c}\right)$. In Figure 3, there are two interesting situations. When there is a unique steady state it is the unique equilibrium. When there are two steady states $q_{L}^{e}>0$ and $q_{H}^{e}>q_{L}^{e}$, we have a continuum of equilibrium where $q_{t} \rightarrow q_{H}^{e}$ from above or below, depending on where we start. Notice that in Case 3 it is $q_{H}^{e}$ that is attractive, while $q_{L}^{e}$ is attractive in Case 1.

We summarize these results as follows:

Proposition 1 In $S T W$, for high, intermediate and low $\theta$, the sets of perfect foresight equilibria are described in the discussions of Cases 1, 2 and 3, resp. For high $\theta$, if $\delta<0$ is not too big there are 2 steady states, $q_{L}^{e} \in(0, \bar{q})$ and $q_{H}^{e} \in\left(q_{L}^{e}, \bar{q}\right)$, plus equilibria where $q \rightarrow q_{L}^{e}$. For low $\theta$, if $\delta>0$ is not too big there are 2 steady states $q_{L}^{e} \in(0, \bar{q})$ and $q_{H}^{e} \in\left(q_{L}^{e}, \bar{q}\right)$, plus equilibria where $q \rightarrow q_{H}^{e}$. For intermediate $\theta$, or more extreme $\delta$, there is at most one equilibrium with trade. If $\delta>0$ is too big trade shuts down and agents hoard assets. If $\delta<0$ is too big agents dispose of assets.

Now consider sunspot equilibria, where $q$ fluctuates randomly over time, even though fundamentals are constant. There is random variable $s \in\{1,2\}$, and when $s=$ $i$ it switches to $j \neq i$ according to a Poisson process with arrival rate $\varepsilon_{i}$, independent of everything else in the economy. In a stationary sunspot equilibria, $q_{s}$ is a timeinvariant functions of $s$. With a slight abuse of notation, let the superscript in $V_{m}^{s}$ 
now indicate the sunspot state (not an agent's $\delta$ ), and rewrite the Bellman equations when $s=i$ as

$$
\begin{aligned}
& r V_{1}^{i}=\sigma(1-M)\left[u\left(q_{i}\right)+V_{0}^{i}-V_{1}^{i}\right]+\delta+\varepsilon_{i}\left(V_{1}^{j}-V_{1}^{i}\right) \\
& r V_{0}^{i}=\sigma M\left[-c\left(q_{i}\right)+V_{1}^{i}-V_{0}^{i}\right]+\varepsilon_{i}\left(V_{0}^{j}-V_{0}^{i}\right) .
\end{aligned}
$$

A proper sunspot equilibrium is a pair $\left(q_{1}, q_{2}\right)$, with $q_{1} \neq q_{2}$, solving $g\left(q_{i}\right)=\Delta_{i}$, where $\Delta_{i}$ comes from (14)-(15), for $i=1,2$. Such equilibria formalize the notion of excess volatility in asset values. The following Proposition, proved in Appendix B, verifies they exist. ${ }^{7}$

Proposition 2 In $S T W$ with $\theta \in\left(\theta_{u}, 1\right]$, if there are two steady states $q_{L}^{e} \in(0, \bar{q})$ and $q_{H}^{e} \in\left(q_{L}^{e}, \bar{q}\right)$, for any $q_{1} \in\left(0, q_{L}^{e}\right)$ and $q_{2} \in\left(q_{L}^{e}, q_{H}^{e}\right)$ there $i s$ a sunspot equilibrium where $q$ fluctuates between $q_{1}$ and $q_{2}$. And with $\theta \in\left[0, \theta_{c}\right)$, if there are two steady states $q_{L}^{e} \in(0, \bar{q})$ and $q_{H}^{e} \in\left(q_{L}^{e}, \bar{q}\right)$, for any $q_{1} \in\left(q_{L}^{e}, q_{H}^{e}\right)$ and $q_{2} \in\left(q_{H}^{e}, \bar{q}\right)$ there is a sunspot equilibrium where $q$ fluctuates between $q_{1}$ and $q_{2}$.

This completes the analysis of dynamics. The bottom line is that once one takes seriously the role of assets in facilitating transactions, outcomes can be complicated in interesting ways by the self-referential nature of liquidity. Obviously for $\delta=0$ (fiat money), but also for $\delta>0$, there are equilibria where to get an asset agents pay a cost $c(q)$ above its fundamental value $\delta / r$. Hence, one might say these equilibria display bubbles in a standard sense: "if the reason that the price is high today is only because investors believe that the selling price is high tomorrow - when 'fundamental' factors do not seem to justify such a price - then a bubble exists" (Stiglitz 1990). Now, some may argue that the liquidity service provided by an asset is a fundamental

\footnotetext{
${ }^{7}$ Sunspots in STW are also studied by Shi $(1995)$ and Ennis $(2001,2004)$, using different methods. They also seek a solution to the equilibrium conditions with $q_{1} \neq q_{2}$, but proceed by noting that for $\varepsilon_{1}=\varepsilon_{2}=0$ this is the same as the existence of two steady states. By continuity, if two steady states $q_{L}^{e}$ and $q_{H}^{e}$ exist, for small $\varepsilon_{1}, \varepsilon_{2}>0$ there is a sunspot equilibrium with $q_{1}$ close to $q_{L}^{e}$ and $q_{2}$ close to $q_{H}^{e}$. We instead construct sunspot equilibria around $q_{L}^{e}$ in one case, and around $q_{H}^{e}$ in the other, for any $q_{1}$ and $q_{2}$ in the ranges given in Proposition 2, not merely $q_{i}$ close to steady state.
} 
factor; rather than debate semantic issues, we emphasize the economics: the model can display deterministic and stochastic time-varying equilibria even when fundamentals - preferences, technologies etc. - are constant. ${ }^{8}$
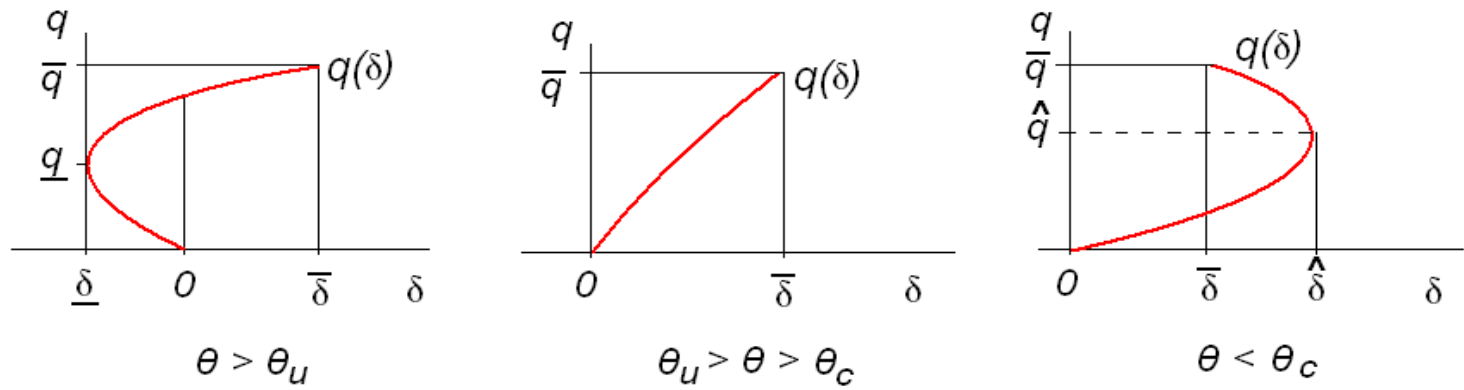

Figure 4: Equilibrium $q$ as a function of $\delta$

Consider now the effects of parameter changes, which are sharper here than in previous analyses. The equilibrium function $e(q)$ shifts with parameters as follows:

$$
\frac{\partial e(\cdot)}{\partial \delta}<0, \frac{\partial e(\cdot)}{\partial r}>0, \frac{\partial e(\cdot)}{\partial M}>0, \frac{\partial e(\cdot)}{\partial \theta}<0, \frac{\partial e(\cdot)}{\partial \sigma} \approx M-\theta .
$$

From (16) one can derive the effects on $q$ simply by shifting the curves in Figures 1-3. Figure 4 illustrates the effects of $\delta$ for different $\theta$, including some situations with multiplicity. Notice that if $\in(0, \bar{\delta})$ there is always a unique steady state and $\partial q / \partial \delta>0$. While multiplicity is interesting, it is also good to know conditions for uniqueness. In any case, we summarize these comparative static results as follows:

Proposition 3 In STW the effects of all parameters on q are determined by (16) and Figures 1-3.

In terms of welfare, let $r W=\delta M+\sigma M(1-M)[u(q)-c(q)]$. Clearly $W$ is maximized wrt $q$ at $q^{*}$. From this and (16) one can study the effects of parameters on

\footnotetext{
${ }^{8}$ More can be done. Coles and Wright (1998), e.g., construct continuous-time cycles where $q$ and $\Delta$ revolve around steady state. That result requires strategic bargaining, however, and one has to work in $\mathbb{R}^{2}$, while here we can eliminate $\Delta=g(q)$ and work in $\mathbb{R}^{1}$. Also, while the results in Coles and Wright might make one question the use of axiomatic bargaining in dynamic models, if $\theta=1$ that point is moot, and this model can generate interesting dynamics with $\theta=1$. Still, it may be interesting to explore dyanmics with alternative strategic bargaining solutions in future work.
} 
$W$. One can also ask when $q^{*}$ obtains in equilibrium. With $\delta=0$, it is easily checked that $q=q^{*}$ is a steady state iff

$$
\theta=\theta^{*} \equiv \frac{r u\left(q^{*}\right)+\sigma M\left[u\left(q^{*}\right)-c\left(q^{*}\right)\right]}{(r+\sigma)\left[u\left(q^{*}\right)-c\left(q^{*}\right)\right]}
$$

Notice $\theta^{*} \leq 1$ iff $r \leq r^{*}$ for some $r^{*}>0$. So, if agents are relatively patient we can achieve the first best $q^{*}$ with $\theta=\theta^{*} \leq 1$; otherwise we cannot, and the second best is achieved at $\theta=1 .^{9}$

To close this Section, we note that in STW agents may want to trade using lotteries. ${ }^{10}$ It is easy to show that we can restrict attention to a deterministic $q$ and a lottery over the asset transfer $d \in\{0,1\}$, say $\pi=\operatorname{prob}(d=1)$. Then we have

$$
\begin{gathered}
r V_{1}=\sigma(1-M)[u(q)-\pi \Delta]+\delta \\
r V_{0}=\sigma M[\pi \Delta-c(q)] \\
\Rightarrow(r+\sigma \pi) \Delta=\delta+\sigma(1-M) u(q)+\sigma M c(q) .
\end{gathered}
$$

Kalai bargaining with lotteries reduces to

$$
\max _{\pi, q}\{u(q)-\pi \Delta\} \text { st } \pi \Delta=g(q) \text { and } \pi \in[0,1] .
$$

If there is trade then $\pi>0$. If $\pi \leq 1$ is binding then $q$ solves $(1-\theta) u(q)+c(q)=$ $\Delta$, exactly as in the economy without lotteries. So, suppose $\pi \leq 1$ is not binding. Then (18) implies $q=q^{*}$ and $\pi=\pi^{*}$, where

$$
\pi^{*} \Delta=(1-\theta) u\left(q^{*}\right)+\theta c\left(q^{*}\right) .
$$

\footnotetext{
${ }^{9}$ This is a version of the Hosios (1990) condition for efficiency in search-and-bargaining models. One can also compute the dividend $\delta^{*}=A_{u} u\left(q^{*}\right)-A_{c} c\left(q^{*}\right)$ that delivers $q^{*}$ for a fixed $\theta$. Or, one can maximize $W$ wrt $M$, which makes sense at least for fiat currency, which can be produced at next-to-no cost. If $q$ were fixed, the solution is $M=1 / 2$, which maximizes trade volume (a special case of Berentsen 2002, who shows that when the upper bound on currency holdings is any integer $\bar{m}$, trade volume is maximized at $M=\bar{m} / 2$ ). But $q$ is endogenous in this model. Suppose there is a unique steady state, where $\partial q / \partial M<0$ (i.e., the nominal price level $1 / q$ is increasing in the money supply). If $\theta<\theta^{*}$, and hence $q<q^{*}$, it is desirable to reduce $M$ below $1 / 2$ because the fall in volume has only a second order cost, by the envelope theorem, while the increase in $q$ has a first-order effect. This formalizes in a stylized way the notion that monetary policy ought to try and balance liquidity provision and inflation.

${ }^{10}$ See, e.g., Berentsen et al. (2002). Generally, a lottery is a probability distribution over the space of feasible trades, $(q, d) \in \mathbb{R}_{+} \times\{0,1\}$, where $q$ is output and $d$ is the payment (asset transfer). Since $m$ is indivisible, this space is not convex and randomization can be useful.
} 
Combining (17) and (19), we arrive at

$$
\pi^{*}=\frac{r\left[(1-\theta) u\left(q^{*}\right)+\theta c\left(q^{*}\right)\right]}{\delta+\sigma(\theta-M)\left[u\left(q^{*}\right)-c\left(q^{*}\right)\right]} .
$$

It is easy to check $\pi^{*} \in(0,1)$ iff $e\left(q^{*}\right)<0$. Referring back to Figures 1 -3, we conclude that whenever $q>q^{*}$ in the economy without lotteries, the equilibrium with lotteries is $q=q^{*}$ and $\pi=\pi^{*}<1$, as shown in Figure 5 .
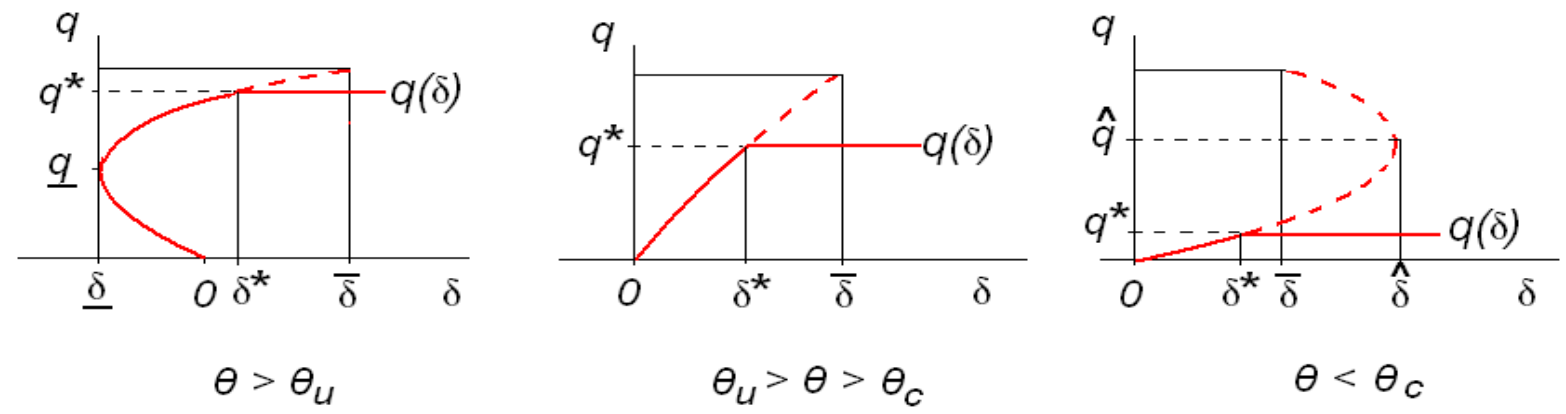

Figure 5: Equilibrium $q$ as a funciton of $\delta$ with lotteries

Comparing Figures 4 and 5, lotteries truncate $q$ by $q^{*}$ : there is a critical $\delta^{*}$ such that $q=q^{*} \forall \delta \geq \delta^{*}$. As $\delta$ increases above $\delta^{*}, \pi^{*}$ falls, and $\pi^{*} \rightarrow 0$ as $\delta \rightarrow \infty$. Trade does not cease when $\delta$ gets big: the buyer always gets $q=q^{*}$, but the asset changes hands with lower and lower probability. So, Gresham's Law does not hold in the sense that the circulation of $m$ stops, but it certainly slows down. Also, note that we lose the multiplicity we had in Case 3 when there were no lotteries. We maintain multiplicity in Case 1 , however, where for $\underline{\delta}<\delta<0$ there are two steady states with $q<q^{*}$. Hence, in Case 1, there are dynamic equilibria where $q \rightarrow q_{L}^{e}$ from above or below depending on where we start. So lotteries change some details, but the salient results survive.

Some of the above results are new relative to previous analyses of STW. Others generalize or simplify the proofs of known results, although in a sense everything is new, as previous analyses used Nash, rather than Kalai bargaining. While more can be done, we now turn to financial economics. 


\section{Finance}

Assume $u(q)=c(q)=q$ and $\delta_{H}>\delta_{L}$, so there are no gains from trading $q$ but there are gains from trading $m$. A moment's reflection leads to the conclusion that trade occurs iff an agent with $\delta_{H}$ and $m=0$ meets one with $\delta_{L}$ and $M=1$, so $\phi_{L H}=1$ and $\phi_{H H}=\phi_{L L}=\phi_{H L}=0$. Then (1)-(4) reduce to a quadratic equation one can solve for steady state. To ease notation, normalize $\sigma=1,{ }^{11}$ and let $\gamma_{H}=\lambda_{L} /\left(\lambda_{L}+\lambda_{H}\right)$ and $\gamma_{L}=\lambda_{H} /\left(\lambda_{L}+\lambda_{H}\right)$ be the (unconditional) proportions of type $H$ and $L$. Then

$$
\begin{aligned}
n_{0}^{H} & =\frac{1}{2}\left[\gamma_{H}-M-\lambda_{L}-\lambda_{H}+\sqrt{D}\right] \\
n_{1}^{L} & =\frac{1}{2}\left[-\gamma_{H}+M-\lambda_{L}-\lambda_{H}+\sqrt{D}\right] \\
n_{1}^{H} & =\frac{1}{2}\left[\gamma_{H}+M+\lambda_{L}+\lambda_{H}-\sqrt{D}\right] \\
n_{0}^{L} & =\frac{1}{2}\left[2-\gamma_{H}-M+\lambda_{L}+\lambda_{H}-\sqrt{D}\right]
\end{aligned}
$$

is the steady state, where $D=\left(\gamma_{H}-M+\lambda_{L}+\lambda_{H}\right)^{2}+4 \lambda_{H} M$. The key variables are $n_{0}^{H}$ and $n_{1}^{L}$, measuring effective demand and supply, and their product $n_{1}^{L} n_{0}^{H}$, measuring trade volume.

As a special case of (5)-(8), Bellman's equations become

$$
\begin{aligned}
& r V_{1}^{H}=\delta_{H}+\lambda_{H}\left(V_{1}^{L}-V_{1}^{H}\right)+\dot{V}_{1}^{H} \\
& r V_{0}^{H}=\lambda_{H}\left(V_{0}^{L}-V_{0}^{H}\right)+n_{1}^{L}\left(V_{1}^{H}-V_{0}^{H}-q\right)+\dot{V}_{0}^{H} \\
& r V_{1}^{L}=\delta_{L}+\lambda_{L}\left(V_{1}^{H}-V_{1}^{L}\right)+n_{0}^{H}\left(q+V_{0}^{L}-V_{1}^{L}\right)+\dot{V}_{1}^{L} \\
& r V_{0}^{L}=\lambda_{L}\left(V_{0}^{H}-V_{0}^{L}\right)+\dot{V}_{0}^{L},
\end{aligned}
$$

where $q=q_{L H}$ is what an agent with $\delta_{L}$ gets for his asset from one with $\delta_{H}$. As indicated by (25), a high valuation agent with $m=1$ enjoys the flow $\delta_{H}$ until he switches to $\delta_{L}$. After a switch, as indicated by (27), he enjoys the reduced flow $\delta_{L}$, and either switches back or trades the asset away, whichever comes first.

\footnotetext{
${ }^{11}$ Recall $\sigma$ is the product of contact rate $\alpha$ and the probability of a single coincidence $1 / K$; but we do not actually need $K>1$ in DGP.
} 
As regards the terms of trade, now Kalai bargaining yields

$$
q=\theta \Delta^{H}+(1-\theta) \Delta^{L}
$$

which is the same as Nash because utility is linear in DGP. It is now a matter of solving (25)-(28) for $\Delta^{j}=V_{1}^{j}-V_{0}^{j}$, inserting these into (29) and simplifying to get

$$
r q=\eta \delta_{H}+(1-\eta) \delta_{L}
$$

which gives $q$ as a weighted average of the two fundamental valuations of the dividend, with weight $\eta=\left(\lambda_{L}+\theta r+\theta n_{0}^{H}\right) / C$, and it is convenient to define

$$
C \equiv r+\lambda_{L}+\lambda_{H}+\theta^{H} n_{0}+(1-\theta) n_{1}^{L}
$$

This construction delivers the unique steady state. We claim that it is a saddle point. Therefore, starting at any initial $\mathbf{n}_{0}$, there is a unique path that neither explodes nor becomes negative - the saddle path converging to steady state. Here is the argument: First, subtracting (25)-(26) and (27)-(28), after eliminating $q$ using (29), we get two differential equations in $\Delta^{H}$ and $\Delta^{L}$. Then, as always, using the identities $n_{0}^{L}+n_{0}^{H}=1-M$ and $n_{1}^{L}+n_{1}^{H}=M$, we get two differential equations in $n_{0}^{H}$ and $n_{1}^{L}$. This yields a $4 \times 4$ dynamical system:

$$
\begin{aligned}
\dot{\Delta}_{H} & =r \Delta_{H}-\delta_{H}+\lambda_{H}\left(\Delta_{H}-\Delta_{L}\right)+n_{1}^{L}(1-\theta)\left(\Delta_{H}-\Delta_{L}\right) \\
\dot{\Delta}_{L} & =r \Delta_{L}-\delta_{L}-\lambda_{L}\left(\Delta_{H}-\Delta_{L}\right)-n_{0}^{H} \theta\left(\Delta_{H}-\Delta_{L}\right) \\
\dot{n}_{0}^{H} & =\lambda_{L}\left(1-M-n_{0}^{H}\right)-\lambda_{H} n_{0}^{H}-n_{0}^{H} n_{1}^{L} \\
\dot{n}_{1}^{L} & =\lambda_{H}\left(M-n_{1}^{L}\right)-\lambda_{L} n_{1}^{L}-n_{1}^{L} n_{0}^{H}
\end{aligned}
$$

Appendix F calculates the eigenvalues for system (32)-(35) analytically and verifies saddle-path stability. ${ }^{12}$

\footnotetext{
${ }^{12}$ This is a new result. As we said concerning Lemma 1, Duffie et al. (2005) show that the system for $\mathbf{n}(1)-(4)$ is stable. We show the condition for determinacy (uniqueness) of equilibrium, not just the dynamic system (1)-(4). This involves showing the joint system $(\mathbf{n}, \mathbf{V}, q)$ displays saddle point stability, which we do after reducing it to a $4 \times 4$ system, as described in the text.
} 
Lemma 2 Dynamical system (32)-(35) has two positive and negative eigenvalues.

Hence, equilibrium (not only steady state) is unique: starting from any initial conditions there is a unique transition to the steady state. We return to transition momentarily, after discussing steady states. First, we have $\partial q / \partial \delta_{H}>0$ and $\partial q / \partial \delta_{L}>$ 0 , so making the asset better as a store of value increases its worth when trading for q. Also, naturally, $q$ is decreasing in $r$ and increasing in $\theta$. Moreover, $q$ is increasing in $n_{0}^{H}$ (effective asset demand) and decreasing in $n_{1}^{L}$ (effective asset supply). Of course, $n_{0}^{H}$ and $n_{1}^{L}$ are endogenous, but one can check that $n_{0}^{H}$ falls and $n_{1}^{L}$ rises with $M$, so $\partial q / \partial M<0$. In terms of the switching process, consider changing $\lambda_{L}$ or $\lambda_{H}$ holding $n_{0}^{H}$ and $n_{1}^{L}$ constant, say, by adjusting $M$ and $\sigma$. Then $q$ rises with $\lambda_{L}$ and falls with $\lambda_{H}$. If we do not hold $n_{0}^{H}$ and $n_{1}^{L}$ constant, one can still show $q$ rises with $\lambda_{L}$ and falls with $\lambda_{H}$ using a result proved in Appendix C.

Lemma $3 \partial n_{0}^{H} / \partial \lambda_{L}>0, \partial n_{0}^{H} / \partial \lambda_{H}<0, \partial n_{1}^{L} / \partial \lambda_{L}<0$ and $\partial n_{1}^{L} / \partial \lambda_{H}>0$.

We summarize these results as:

Proposition 4 In DGP there exists a unique equilibrium from any initial condition $\mathbf{n}_{0}$, and a unique steady state, where $\mathbf{n}$ is given by (21)-(24) and $q$ by (29).

Proposition 5 In DGP the effects of parameters on $q$ are: $\partial q / \partial \delta_{j}>0, \partial q / \partial r<0$, $\partial q / \partial \theta>0, \partial q / \partial M<0, \partial q / \partial \lambda_{L}>0$ and $\partial q / \partial \lambda_{H}<0$.

A main goal of the paper is to contrast these results for with those in Section 3. The next Proposition describes some technical differences:

Proposition 6 (1) In DGP, there is always a unique equilibrium and a unique steady state; in STW there can be multiple steady states and nonstationary equilibria. (2) In DGP with $\delta_{L}, \delta_{H}>0$, agents always trade when one with $\delta=\delta_{L}$ and $m=1$ meets one with $\delta=\delta_{H}$ and $m=0$; in $S T W$ with $\delta>0$, agents stop trading and hoard assets 
if $|\delta|$ is big. (3) In DGP with $\delta_{L}, \delta_{H}<0$, agents stop trading and dispose of assets; in $S T W$ with $\delta<0$, there can be trade as long as $|\delta|$ is not too big.

Another difference is that, since DGP is not a model of fiat money, it is less interesting to analyze welfare $W$ as a function of $M$ (although one still can ask, e.g., how $M$ affects trading volume). Yet another difference it that, when $u(q)=c(q)=$ $q$, one can easily show there is no gain from introducing lotteries. But perhaps the biggest difference between the models, given people in finance are interested in dynamics and volatility arising from self-fulfilling prophecies, is that there is no way in DGP to construct anything like the deterministic or stochastic nonstationary equilibria for STW in Section 3. However, there are interesting transitional dynamics in DGP, resulting from the distribution $\mathbf{n}$ being endogenous, that do not arise in STW. ${ }^{13}$
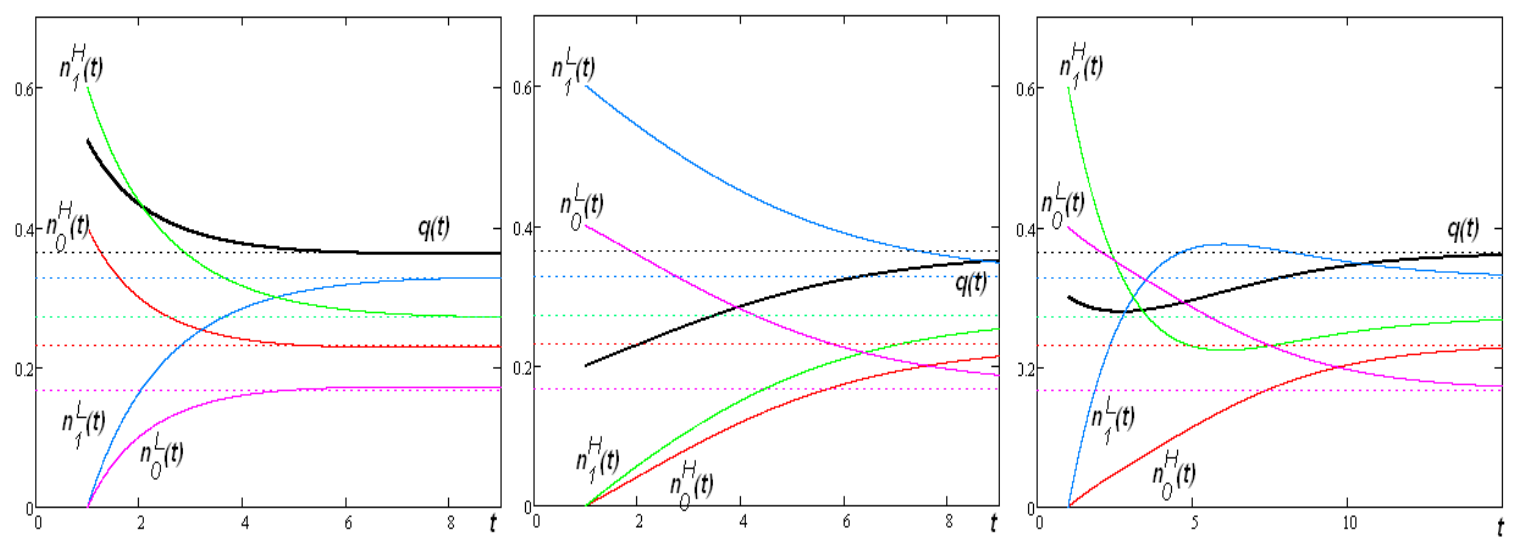

Figure 6: Short-run asset market dynamics

Such transitional dynamics are studied to good effect by Duffie et al. (2007) and Weill $(2007,2008)$, but we construct our own examples. Suppose we start in steady state, but at date $t=1$ we shock $\mathbf{n}$ as a surprise to the agents in the model. As n converges back to steady state, over time, there are perfect-foresight equilibrium

\footnotetext{
${ }^{13}$ In STW, $M$ agents always hold $m=1$ and $1-M$ agents always hold $m=0$, no matter what else happens, short of agents disposing of their assets.
} 
price movements. Figure 6 shows three examples. In the left panel, the shock is a jump in short-run asset demand: at $t=1$ we set $\delta=\delta_{H}$ for all agents. This raises $q$ on impact, after which it decreases monotonically back to steady state. The middle panel is what is sometimes called an aggregate liquidity shock: we set $\delta=\delta_{L}$ for everyone, so they all want to divest themselves of assets. Now $q$ drops on impact before regaining value as we converge back to steady state. The right panel displays the transition after a shock that sets $\delta=\delta_{L}$ for all agents with $m=0$ and $\delta=\delta_{H}$ for all those with $m=1$, so demand and supply both tighten. This generates a nonmonotone path for $q$ and $\mathbf{n}$ as we converge back to steady state.

Importantly, Duffie et al. (2005) also study intermediation. ${ }^{14}$ Assume at rate $\beta$ agents meet third parties called brokers. In these meetings, if the agent is in state $\delta_{H}$ with $m=0$, a broker would like to offer him the asset, asking $q_{a}$ for it; and if the agent is in state $\delta_{L}$ with $m=1$, a broker would like to take the asset off his hands, bidding $q_{b}$ for it. For now brokers do not hold assets, but they buy and sell them in a competitive interdealer market, clearing at each instant by giving 0 surplus to the long side. The long and short sides depend on which is bigger, effective supply $n_{1}^{L}$ or demand $n_{0}^{H}$. One can show $n_{1}^{L}>n_{0}^{H}$ iff $M>\gamma_{H}$. If $n_{1}^{L}>n_{0}^{H}$ there are more agents trying to sell assets to dealers than trying to buy them, so dealers trying to off-load assets are on the long side of the interdealer market and the price is $q_{b}$. In this case, dealers do not take assets from everyone they meet with $m=1$. If $n_{1}^{L}>n_{0}^{H}$ there are fewer agents trying to sell assets to dealers, so dealers trying to off-load them are on the short side of the interdealer market and the price is $q_{a}$. In this case, dealers do not sell assets to everyone with $m=0$.

To do it slightly differently, suppose as an alternative to the interdealer market that each broker manages a collection of automated terminals of sufficient measure

\footnotetext{
${ }^{14}$ Introducing intermediaries into the OTC market is one of the main contributions of DGP. However, search-based models of middlemen go back to Runbinstein and Wolinsky (1987), with Li $(1998,1999)$ and Shevichenko (2004) providing examples in the spirit of STW. In our view, a bigger contribution of DGP was the idea to apply search theory to financial markets in the first place.
} 
to take advantage of the law of large numbers, in the spirit of Shi (1997). There is a fixed cost $\omega$ to setting up these terminals. Agents contact terminals according to a Poisson process with arrival rate $\beta$, which depends in general on the measure of brokers in the market. A broker now does not have to trade on an interdealer market, assuming he can shift assets across terminals, but he still may not trade with every agent he meets in the OTC market - e.g., if $n_{1}^{L}>n_{0}^{H}$ brokers trade with a fraction $n_{0}^{H} / n_{1}^{L}$ of those they meet, which equates their asset inflow and outflow.

For the sake of illustration, suppose brokers have all the bargaining power with their customers - terminals are programmed with $q_{b}$ and $q_{a}$ to extract all gains from trade. It is then easy to verify the following:

Proposition 7 In DGP, with intermediaries that have all the bargaining power, the bid and ask prices satisfy

$$
r q_{b}=\eta_{b} \delta_{H}+\left(1-\eta_{b}\right) \delta_{L} \text { and } r q_{a}=\eta_{a} \delta_{H}+\left(1-\eta_{a}\right) \delta_{L}
$$

where the weights are given by $\eta_{b}=\left(\lambda_{L}+\theta n_{0}^{H}\right) / C$ and $\eta_{a}=\left(r+\lambda_{L}+\theta n_{0}^{H}\right) / C$ with $C$ defined in (31). The bid-ask spread is $q_{b}-q_{a}=\left(\delta_{H}-\delta_{L}\right) / r C>0$. When agents trade with each other $q$ still satisfies (30), with $q \in\left(q_{a}, q_{b}\right)$ if $\theta \in(0,1)$.

A positive bid-ask spread means dealers make profits. Clearly, the spread is increasing in $\delta_{H}$, decreasing in $\delta_{L}$, and vanishing as $\delta_{H}-\delta_{L} \rightarrow 0$. This last result indicates why there is no role for this kind of intermediation in the model of Section 3 , where $\delta_{H}=\delta_{L}$, which is another significant distinction between DGP and STW. ${ }^{15}$

Consider $n_{1}^{L} \geq n_{0}^{H}$ (the other case is similar). Appendix D solves for steady state with brokers, generalizing (21)-(24). Then intermediary profit is

$$
\Pi=n_{0}^{H}\left(\delta_{H}-\delta_{L}\right) / C-\omega
$$

\footnotetext{
${ }^{15}$ At least, there is no role for intermediaries in the baseline STW model presented above. Their role in the papers mentioned fn. 14 arises from middlemen having a superior search technology, informational advantages, or the ability to hold large inventories.
} 
which nets out the cost $\omega$. Suppose we allow free entry. Then the number of intermediaries adjusts until $\Pi=0$, which implies

$$
n_{0}^{H}=\frac{\omega\left[r+\lambda_{L}+\lambda_{H}-(1-\theta)\left(M-\gamma_{H}\right)\right]}{\delta_{H}-\delta_{L}-\omega} .
$$

Alternatively, suppose we have a monopoly broker, who chooses his number of terminals to maximize $\Pi$ knowing that his choice affects his arrival rates. One can then easily compare competitive and monopoly intermediation.

Instead of pursuing that exercise, consider a new application. Suppose intermediaries can hold or issue assets that others consider perfect substitutes for the assets already in the model. We call these intermediaries banks (it is just a label). Let $N$ be the net asset issuance by banks. If $N>0$ the nonbank public is holding bank assets; if $N<0$ then the bank is holding nonbank assets. We call $M$ and $N$ outside and inside assets, following Holmstrom and Tirole (2011). A bank's return from holding assets is $-N \delta_{B}$, since $N>0$ means the bank issues assets, where one can set $\delta_{B}$ as one likes, e.g., as an average of $\delta_{H}$ and $\delta_{L}$. For the sake of illustration, assume a bank must trade with any agent it meets, as might be reasonable at least for central banks. Now $N$ is endogenously determined so that $n_{1}^{L}=n_{0}^{H}$. The other steady state conditions are the same as before, except $n_{1}^{H}+n_{1}^{L}=M+N$ (previously $N=0$ ). Appendix D solves for steady state, from which the following is immediate:

Proposition 8 In DGP with banking, any increase in outside assets $M$ is completely crowded out by an endogenous change in inside assets $N$, so that the total steady-state asset supply is always $M+N=\gamma_{H}$.

We read this Proposition as saying asset demand creates its own supply - finance's version of Say's Law. It is a new result, we think, in the context of DGP, although there are related ideas in the literature (e.g., Burdett et al. 2001). One can potentially do more with this model. For instance, bank profit is

$$
\Pi=\left(M-\gamma_{H}\right) \delta_{H}+n_{0}^{H}\left(\delta_{H}-\delta_{L}\right) /\left(r+\lambda_{L}+\lambda_{H}+n_{0}^{H}\right)-\omega
$$


With free entry, steady state adjusts until $\Pi=0$, from which one can determine $n_{0}^{H}$. One can then analyze when equilibrium delivers an efficient number of banks. Rather than pursue extensions of these models, we move to some more novel material.

\section{General Equilibrium}

The theory presented above is GE (general equilibrium) in the same sense that, say, Mortensen and Pissarides (1994) is a GE theory of the labor market: it is internally consistent, agents maximize, quantities and prices are endogenous, and expectations are rational. But these are not very general equilibrium models, in the sense that our OTC market, like the labor market in Mortensen and Pissarides, is the only venue for economic activity. Here we provide a simple way to embed the OTC model in GE with minimal deviation from the benchmark setup, and in particular while continuing to assume $m \in\{0,1\}$. For this it is convenient to switch to discrete time, and have agents alternate between trading in an OTC market and a Walrasian market. ${ }^{16}$

To begin, consider $\delta_{i}=\delta$ as in STW, and for now focus on stationary equilibrium. The discrete-time Bellman equations in the OTC market are

$$
\begin{aligned}
V_{1} & =\sigma(1-M)\left[u(q)+W_{0}\right]+[1-\sigma(1-M)] W_{1} \\
V_{0} & =\sigma M\left[-c(q)+W_{1}\right]+(1-\sigma M) W_{0},
\end{aligned}
$$

where $W_{m}$ is the value function entering the Walrasian market,

$$
W_{m}=\max \left\{U(x)-\ell+(1+r)^{-1} V_{m^{\prime}}\right\} \text { st } x=w \ell+(\delta+p) m-p m^{\prime}, m^{\prime} \in\{0,1\} .
$$

Here $U(x)$ is the utility of consuming a good $x$, which we use as numeraire, $\ell$ is labor supply, $w$ is the wage and $p$ is the asset price. Notice the dividend $\delta$ now arrives

\footnotetext{
${ }^{16}$ Following Lagos and Wright (2005), the standard New Monetarist model discussed in the surveys mentioned in fn. 1 also has agents switching between decentralized and centralized trade in discrete time. This device has previously been used to embed the Mortensen-Pissarides labor market in GE by Berentsen et al.(2011) and Bethune et al.(2013). There are also continuous time versions (e.g., Craig and Rocheteau 2008; Rodriguez-Lopez and Rocheteau 2013; Rocheteau and Wong 2013), but the discrete time version seems (we think) more natural.
} 
in the Walrasian market in units of numeraire. For ease of exposition, assume $x$ is produced one-for-one with $\ell$, so the equilibrium wage is $w=1 .^{17}$

Using the budget equation to eliminate $\ell$, we get

$$
W_{m}=\max \left\{U(x)-x+(\delta+p) m-p m^{\prime}+(1+r)^{-1} V_{m^{\prime}}\right\} \text { st } m^{\prime} \in\{0,1\} .
$$

This yields $x=x^{*}$, where $U^{\prime}\left(x^{*}\right)=1$, and $W_{1}-W_{0}=\delta+p$. Given $M \in(0,1)$, in equilibrium some agents choose to be consumers in the OTC market with $m^{\prime}=1$, while others choose to be producers with $m^{\prime}=0$, and they are indifferent: $(1+r) p=$ $V_{1}-V_{0}$. Using (36)-(37), we get the Walrasian asset-pricing condition

$$
(r+\sigma) p=\sigma(1-M) u(q)+\sigma M c(q)+(1-\sigma) \delta
$$

There is a different condition in the OTC market, determined by bargaining as before, but instead of $g(q)=V_{1}-V_{0}$ we have $g(q)=W_{1}-W_{0}$. This boils down to

$$
0=\tilde{e}(q) \equiv A_{u} u(q)-A_{c} c(q)-(1+r) \delta
$$

Steady state is given by $q$ satisfying (40), $p$ satisfying (39), $x=x^{*}$ and $\ell$ satisfying the budget equation. Clearly $\tilde{e}(q)$ is the same as $e(q)$ in Section 3, except $\tilde{\delta}=\delta(1+r)$ replaces $\delta$, since when one acquires an asset in the OTC market, now one has to wait a period for dividends. We can use the properties of $e(q)$ derived above to immediately establish several results: $\forall \tilde{\delta} \in(0, \bar{\delta})$ there is a unique equilibrium; if $\tilde{\delta}=0$ there are two steady states plus dynamic equilibria where $q \rightarrow 0$ and $p \rightarrow 0$; for $\tilde{\delta} \in(0, \underline{\delta})$ and big $\theta$ or $\tilde{\delta} \in(\bar{\delta}, \hat{\delta})$ and small $\theta$, there are multiple steady states, plus dynamic and sunspot equilibria where $q$ and $p$ vary for no fundamental reason. ${ }^{18}$

The structure here makes equilibrium simple, but also special, in that $x$ are $q$ independent. One way to generalize this is to relax the separability between $q$ and $x$

\footnotetext{
${ }^{17}$ It is easy to introduce a concave technology $f(\ell)$, add capital, incorporate taxes, allow many goods, etc. Also, notice there is discounting betwen the Walrasian and OTC markets, but not between the OTC and Walrasian markets; this is merely to reduce notation. It simplfies some calculations to have $\ell$ enter utility linearly, but it is not otherwise important.

${ }^{18} \mathrm{~A}$ minor technicality is that in discrete time the sunspot transition probabilities have to satisfy $\varepsilon_{1}, \varepsilon_{2} \in[0,1]$, while in continuous time we only need $\varepsilon_{1}, \varepsilon_{2}>0$.
} 
in utility. To this end, let agents acquire $x$ in the Walrasian market but only consume it after OTC trading. Thus, in the Walrasian market they choose either $m^{\prime}=1$ and $x=x_{1}$ or $m^{\prime}=0$ and $x=x_{0}$, taking into account the utility of consuming $x_{1}$ and $q$ is $u\left(q, x_{1}\right)$, while the utility of consuming $x_{0}$ and producing $q$ is $v\left(q, x_{0}\right)$. In Appendix E, we argue the following: under reasonable conditions, $q$ increases with $\delta$. If $u_{x q}>0$ then $x_{1}$ also increases with $\delta$, and if $v_{x q}>0$ so does $x_{0}$. So when $q$ and $x$ are complements, $x_{1}$ and $x_{0}$ increase with dividends. So does employment $\ell$, and welfare $W$. Good times on Wall Street mean good times on Main Street. ${ }^{19}$

We can similarly embed the baseline DGP model in GE. Assume the shock to $\delta$ occurs after one enters the OTC market but before trading. Then the discrete-time Bellman equations at the opening of the OTC market are:

$$
\begin{aligned}
V_{1}^{H} & =\left(1-\lambda_{H}\right) W_{1}^{H}+\lambda_{H}\left[\sigma n_{0}^{H}\left(q+W_{0}^{L}\right)+\left(1-\sigma n_{0}^{H}\right) W_{1}^{L}\right] \\
V_{0}^{H} & =\lambda_{L} W_{0}^{L}+\left(1-\lambda_{H}\right)\left[\sigma n_{1}^{L}\left(W_{1}^{H}-q\right)+\left(1-\sigma n_{0}^{H}\right) W_{0}^{H}\right] \\
V_{1}^{L} & =\lambda_{L} W_{1}^{H}+\left(1-\lambda_{L}\right)\left[\sigma n_{0}^{H}\left(q+W_{0}^{L}\right)+\left(1-\sigma n_{0}^{H}\right) W_{1}^{L}\right] \\
V_{0}^{L} & =\left(1-\lambda_{L}\right) W_{0}^{L}+\lambda_{L}\left[\sigma n_{1}^{L}\left(W_{1}^{H}-q\right)+\left(1-\sigma n_{0}^{H}\right) W_{0}^{H}\right]
\end{aligned}
$$

The first says that if one starts the OTC market with $\delta_{H}$ and $m=1$ and $\delta$ does not switch, one holds onto $m$; but if $\delta$ switches one tries to trade the asset. And similarly for the rest.

In the Walrasian market, for an agent with $\delta_{i}$ and $m$, we have

$$
W_{m}^{i}=\max \left\{U(x)-x+\left(\delta_{i}+p\right) m-p m^{\prime}+(1+r)^{-1} V_{m^{\prime}}^{i}\right\} \text { st } m^{\prime} \in\{0,1\}
$$

which is the same as (38) except the value functions and dividend are indexed by the asset valuation $i$. The interpretation is slightly different from the baseline model: agents no longer derive a direct payoff from the asset; they derive a payoff by converting returns into purchasing power. So, the asset could be a productive input, and

\footnotetext{
${ }^{19}$ Of course, if $u_{x q}<0$ and $v_{x q}<0$ then $x_{0}, x_{1}$ and $\ell$ fall with $\delta$, although $W$ still rises.
} 
agents have an opportunity to turn it into $\delta_{i}$ units of numeraire. When someone with $m=1$ and $\delta=\delta_{i}$ contacts someone with $m=0$ and $\delta=\delta_{j}>\delta_{i}$ there are gains from reallocating assets to more productive use.

Assume the $\delta$ shocks are i.i.d., with $\zeta=\operatorname{prob}\left(\delta_{i}=\delta_{H}\right)$. Then $V_{m}^{i}=V_{m}$ for everyone at the start of OTC trading, and $n_{1}^{L}=M(1-\zeta)$ and $n_{0}^{H}=(1-M) \zeta$ are effective asset supply and demand in the market. Then

$$
\begin{aligned}
& V_{1}=\zeta W_{1}^{H}+(1-\zeta)\left\{\sigma(1-M) \zeta\left(q+W_{0}^{L}\right)+[1-\sigma(1-M)] W_{1}^{L}\right\} \\
& V_{0}=(1-\zeta) W_{0}^{L}+\zeta\left\{\sigma M(1-\zeta)\left(W_{1}^{H}-q\right)+[1-\sigma M(1-\zeta)] W_{0}^{H}\right\} .
\end{aligned}
$$

The bargaining solution and market clearing determine the OTC and Walrasian value of the asset, resp.,

$$
q=p+\theta \delta_{L}+(1-\theta) \delta_{H} \text { and } r p=\tilde{\eta} \delta_{H}+(1-\tilde{\eta}) \delta_{L}
$$

where $\tilde{\eta}=\zeta[1+(1-\zeta) \sigma(1-\theta-M)]$.

More can be done. For instance, rather than i.i.d., suppose $\delta_{i}$ is persistent. It is no longer the case that everyone is indifferent between bringing $m=0$ and $m=1$ to the OTC market. If $M$ is small, $p$ adjusts until agents with $\delta_{H}$ are indifferent between $m=0$ and $m=1$ while those with $\delta_{L}$ strictly prefer $m=0$. And if $M$ is big, $p$ adjusts until agents with $\delta_{L}$ are indifferent while those with $\delta_{H}$ strictly prefer $m=1$. It is also possible to consider multiple rounds of OTC trade before agents visit the Walrasian market. ${ }^{20}$ Also, once we put DGP into GE, we no longer need assume they trade $q$ physically in the OTC market. Suppose instead that when an agent with $\delta=\delta_{L}$ and $m=1$ meets one with $\delta=\delta_{H}$ and $m=0$, the latter acquires the asset in exchange for a promise to deliver $d$ (for debt) units of purchasing power in the next Walrasian market.

\footnotetext{
${ }^{20}$ This is related to the model Afonso and Lagos (2012) use to study to the federal funds market, and to the model in Berentsen et al. (2005). It should not be difficult to track the distribution of $\mathbf{n}$ over several rounds of OTC trading, as in Figure 6, before the Walrasian market convenes.
} 
Given this alternative scenario, let $W_{m}^{i}(d)$ be the Walrasian value function for an agent with $\delta_{i}, m$ and debt $d$. The OTC Bellman equations satisfy

$$
\begin{aligned}
& V_{1}=\zeta W_{1}^{H}(0)+(1-\zeta)\left\{\sigma(1-M) \zeta W_{0}^{L}(-d)+[1-\sigma(1-M)] W_{1}^{L}(0)\right\} \\
& V_{0}=(1-\zeta) W_{0}^{L}(0)+\zeta\left\{\sigma M(1-\zeta) W_{1}^{H}(d)+[1-\sigma M(1-\zeta)] W_{0}^{H}(0)\right\},
\end{aligned}
$$

since those with $m=0$ who realize $\delta_{H}$ and meet a counterparty acquire the asset and debt $d$, while those those with $m=1$ who realize $\delta_{L}$ and meet a counterparty give up the asset for a credit (negative debt) $-d$. Having $\ell$ enter utility linearly is nice, but not necessary, as it implies $W_{1}^{L}(d)$ is linear, and the bargaining solution reduces to $d=p+\theta \delta_{L}+(1-\theta) \delta_{H}$.

In general, the point is not that the outcomes are so different, but by putting DGP into GE one is lead to different interpretations. The same is true for STW. Thus, one can assume agents get $q$ without transferring the asset, but in exchange for a promise of payment in the next Walrasian market, perhaps collateralized by the asset, as in Kiyotaki and Moore (1997). And it seems interesting to be able to study, e.g., the effects of OTC asset trade on other markets, such as the labor market. While all of this is worth pursuing, again we move to something new.

\section{The Integrated Model}

Consider the general model, where preferences are nonlinear and $\delta$ switches over time. Now an agent with $\delta=\delta_{H}$ may trade away an asset to get $q$, or an agent with $\delta=\delta_{L}$ may accept an asset in exchange for $q$. The goal is to describe the different possible trading patterns that emerge. For arbitrary bargaining power $\theta$, the algebra is complicated, but much insight can be gained from the case $\theta=1$. Also, we assume $\delta_{H} \geq \delta_{L} \geq 0$ here, and normalize (without loss of generality) $c(q)=q$. Then $V_{0}^{H}=V_{0}^{L}=0, q_{H L}=q_{L L}=V_{H}^{L}$ and $q_{H H}=q_{L H}=V_{1}^{H}$. Hence, there are just two $q$ 's to determine, $q_{L}=q_{b L}$ and $q_{H}=q_{b L}$, and it should be clear that $q_{H} \geq q_{L}$ 
with equality only if $\delta_{L}=\delta_{H}$ (an agent with $\delta_{H}$ is always willing to produce more to get the asset than one with $\left.\delta_{L}\right)$.

There are still many candidate equilibria, as each of the four endogenous trading decisions in the vector $\phi=\left(\phi_{L H}, \phi_{L L}, \phi_{H H}, \phi_{H L}\right)$ can take one of three values: always trade $\phi_{b s}=1$; never trade $\phi_{b s}=0$; or $\operatorname{mix} \phi_{b s}=\Phi$. The next result considerably reduces the number of candidates:

Lemma 4 In the integrated model with $\delta_{H}>\delta_{L}>0$, we have: $\phi_{H L}=1 \Rightarrow \phi_{H H}=$ $1 \Rightarrow \phi_{L L}=1 \Rightarrow \phi_{L H}=1$; and $\phi_{L H}=0 \Rightarrow \phi_{L L}=0 \Rightarrow \phi_{H H}=0 \Rightarrow \phi_{H L}=0$.

Proof: Given $q_{H}>q_{L}$, any pair $\left(q_{H}, q_{L}\right)$ satisfying $u\left(q_{L}\right) \geq q_{L}$ also satisfies $u\left(q_{H}\right)>$ $q_{L}$. Therefore $\phi_{L H} \geq \phi_{L L}$. Similarly, if $u\left(q_{H}\right) \geq q_{H}$ then $q_{H}<\bar{q}$, which implies $q_{L}<\bar{q}$ and hence $u\left(q_{L}\right)>q_{L}$. Therefore $\phi_{L L} \geq \phi_{H H}$. And if $u\left(q_{L}\right) \geq q_{H}$ then $u\left(q_{H}\right)>q_{H}$. Therefore $\phi_{H H} \geq \phi_{H L}$. The results follow directly from these inequalities.

Lemma 4 implies the only possible pure-strategy equilibria are

$$
\boldsymbol{\phi}_{0}=(0,0,0,0), \boldsymbol{\phi}_{1}=(1,0,0,0), \boldsymbol{\phi}_{2}=(1,1,0,0), \boldsymbol{\phi}_{3}=(1,1,1,0), \boldsymbol{\phi}_{4}=(1,1,1,1)
$$

where the subscript indicates the number of 1's in the vector (e.g., $\phi_{4}$ entails trade in all 4 possible situations). There are also candidate equilibria that involve mixing across these, $\boldsymbol{\phi}_{01}=(\Phi, 0,0,0), \boldsymbol{\phi}_{12}=(1, \Phi, 0,0), \phi_{23}=(1,1, \Phi, 0)$ and $\boldsymbol{\phi}_{34}=$ $(1,1,1, \Phi)$. The goal is to determine when each of these candidates is an equilibrium. First, since $V_{0}^{H}=V_{0}^{L}=0$, we need only consider two Bellman equations. Using the bargaining solution $V_{1}^{H}=q_{H}$ and $V_{1}^{L}=q_{L}$, these are

$$
\begin{aligned}
r q_{H} & =\sigma n_{0}^{H} \phi_{H H}\left[u\left(q_{H}\right)-q_{H}\right]+\sigma n_{0}^{L} \phi_{H L}\left[u\left(q_{L}\right)-q_{H}\right]+\delta_{H}+\lambda_{H}\left(q_{L}-q_{H}\right) \\
r q_{L} & =\sigma n_{0}^{H} \phi_{L H}\left[u\left(q_{H}\right)-q_{L}\right]+\sigma n_{0}^{L} \phi_{L L}\left[u\left(q_{L}\right)-q_{L}\right]+\delta_{L}+\lambda_{L}\left(q_{H}-q_{L}\right) .
\end{aligned}
$$

Our method involves first considering the (endogenous) q's that are consistent with different types of equilibria. Consider first $\phi_{0}$. Although there is no trade in 


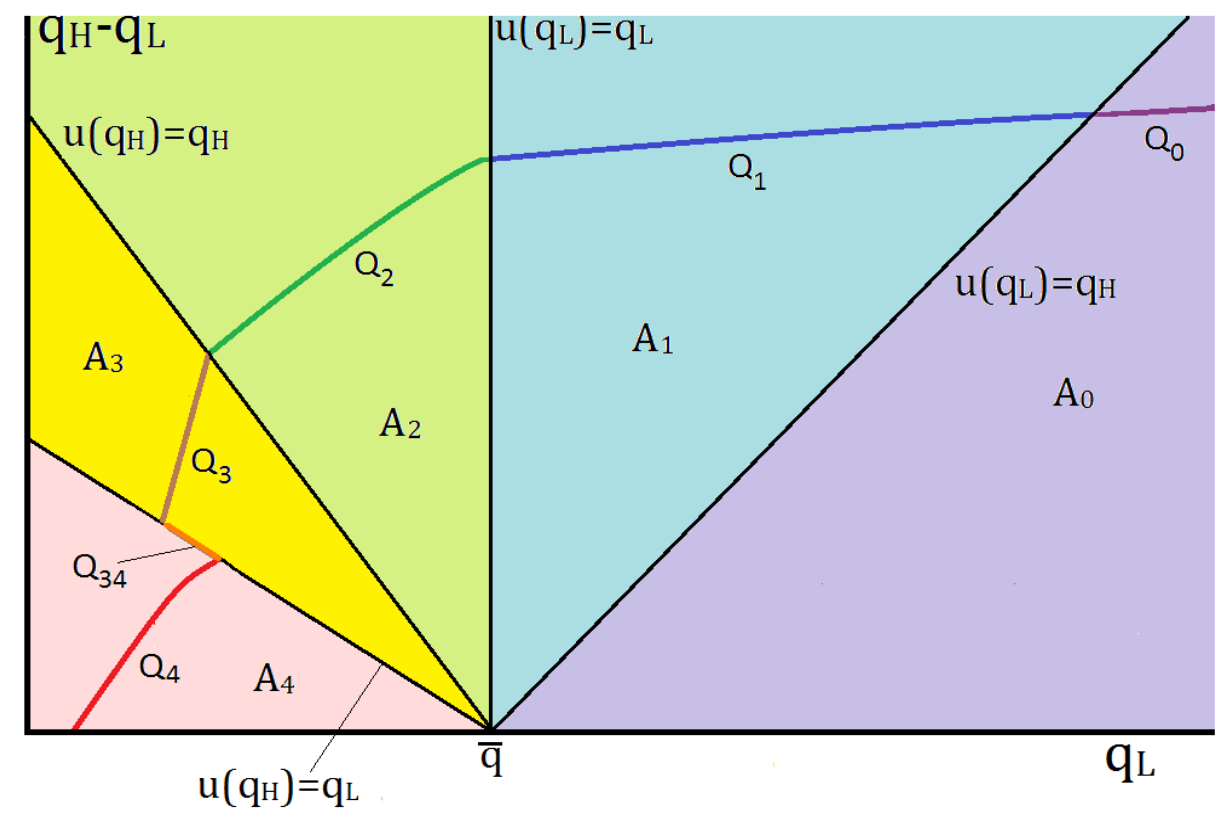

Figure 7: Equilibria in $\left(q_{L}, q_{H}-q_{L}\right)$ space.

this case ${ }^{21}$ obviously, we can still solve for

$$
q_{H}=\frac{\left(r+\lambda_{L}\right) \delta_{H}+\lambda_{H} \delta_{L}}{r\left(r+\lambda_{L}+\lambda_{H}\right)} \text { and } q_{L}=\frac{\left(r+\lambda_{H}\right) \delta_{L}+\lambda_{L} \delta_{H}}{r\left(r+\lambda_{L}+\lambda_{H}\right)} .
$$

For this to be consistent with equilibrium we must check four conditions: $\phi_{L H}=0 \Leftrightarrow$ $u\left(q_{H}\right) \leq q_{L} ; \phi_{L L}=0 \Leftrightarrow u\left(q_{L}\right) \leq q_{L} ; \phi_{H H}=0 \Leftrightarrow u\left(q_{H}\right) \leq q_{H} ;$ and $\phi_{H L}=0 \Leftrightarrow$ $u\left(q_{L}\right) \leq q_{H}$. The last one is the binding condition, by Lemma 4 . Therefore the $\phi_{0}$ equilibrium conditions are satisfied when the $q$ 's given in (43) put us in region $A_{0}$ in Figure 7, with boundary $u\left(q_{L}\right)=q_{H}$.

Now for an important observation. In $\phi_{0}$ equilibrium, where the $q$ 's put us in $A_{0}$, if we reduce $\delta_{H}$ the values of $q_{L}$ and $q_{H}-q_{L}$ both fall. This generates the path shown as $Q_{0}$ in Figure 7. As we continue to reduce $\delta_{H}$, eventually $Q_{0}$ hits the $u\left(q_{L}\right)=q_{H}$ boundary between $A_{0}$ and $A_{1}$ (see below for details). On this boundary the conditions for the $\phi_{0}$ and the $\phi_{1}$ equilibria are satisfied, with (43) giving the $q$ 's in both. Continuing to reduce $\delta_{H}$, we define another path $Q_{1}$, analogous to $Q_{0}$, that goes

\footnotetext{
${ }^{21}$ As in Section 3, the introduction of lotteries may change this outcome to one in which the buyer gets $q^{*}$ and gives up the asset with probability less than 1; we leave this to future work.
} 
through $A_{1}$ until it hits the boundary $u\left(q_{L}\right)=q_{L}$ between $A_{1}$ and $A_{2}$. Equilibrium $\phi_{1}$ is consistent with $q$ 's being in $A_{1}$, where it satisfies the same conditions as $\phi_{0}$ except it reverses the inequality for $\phi_{L H}$, so $u\left(q_{H}\right) \geq q_{L}$, since a buyer with $\delta_{L}$ now buys $q_{L H}$ when he meets a seller with $\delta_{H}$.

If we reduce $\delta_{H}$ further, equilibrium $\phi_{2}$ generates a path $Q_{2}$ starting where $Q_{1}$ hits $u\left(q_{L}\right)=q_{L}$. Again, $Q_{2}$ describes the $q$ 's consistent with the equilibrium conditions for $\phi_{2}$, and in particular, now $u\left(q_{L}\right) \geq q_{L}$, since a buyer with $\delta_{L}$ also buys from a seller with $\delta_{L}$. As we reduce $\delta_{H}$ further, eventually $Q_{2}$ hits $u\left(q_{H}\right)=q_{H}$, at which point we define $Q_{3}$. So $\phi_{2}$ equilibrium is consistent with $q$ 's in $A_{2}$ and $\phi_{3}$ equilibrium with $q$ 's in $A_{3}$. When $Q_{3}$ hits the boundary between $A_{3}$ and $A_{4}$, something different happens: $Q_{3}$ and $Q_{4}$ do meet at this boundary, because $n_{0}^{H}$ and $n_{0}^{L}$ are different in $\phi_{3}$ and $\phi_{4}$. Between $Q_{3}$ and $Q_{4}$ on this boundary we have: if $\phi_{H H}=0$ then $\mathbf{n}$ is such that agents prefer $\phi_{H H}=1$; if $\phi_{H H}=1$ then $\mathbf{n}$ is such that they prefer $\phi_{H H}=0$. Hence, equilibrium consistent with these $q$ 's requires $\phi_{H H} \in(0,1)$, so that $\mathbf{n}$ is such that agents are indifferent between $\phi_{H H}=1$ and $\phi_{H H}=0 .{ }^{22}$ Starting where $Q_{3}$ meets the boundary between $A_{3}$ and $A_{4}$, as we reduce $\delta_{H}, \phi_{H H}$ increases from 0 to 1 , tracing out $Q_{34}$ up to the point where $\phi_{H H}=1$ and $Q_{4}$ intersects the boundary between $A_{3}$ and $A_{4}$. As we continue to reduce $\delta_{H}$ we trace out $Q_{4}$, with $q$ 's consistent with $\phi_{4}$, until we hit $\delta_{H}=\delta_{L}$.

This completes the description of the possible $q$ 's consistent with the different equilibria. To summarize, for a given $\delta_{L}$, if $\delta_{H}$ is very high the equilibrium looks like Gresham's Law, where assets are to valuable to circulate, given concave utility. As $\delta_{H}$ falls, eventually agents that have $m=1$ and $\delta_{L}$ start trading with those that have $m=0$ and $\delta_{H}$, which looks just like the baseline DGP model. As $\delta_{H}$ falls further, eventually agents that have $m=1$ and $\delta_{L}$ start also trading with those that have $m=0$ and $\delta_{L}$. And so on, until $\delta_{H}$ is close to $\delta_{L}$, at which point agents with $m=1$

\footnotetext{
${ }^{22}$ This construction of a mixed-strategy equilibrium does not apply to the other candidate mixed equilibria, because in those candidates $\mathbf{n}$ does not depend on $\phi$.
} 
trade with those who have $m=0$ in every opportunity, and the market looks just like the baseline STW model.

The next step is to translate this characterization of the sets of endogenous q's into sets of exogenous $\delta$ 's that are consistent with different equilibria. This is relatively easy - ones does not have to do much more than label the critical values of $\delta_{H}$, for a given $\delta_{L}$, at which the equilibria switch - once we have the following technical result (the proof, which is simple, is ommitted).

Lemma 5 In the integrated model, $\forall\left(\delta_{L}, \delta_{H}\right)$ we have: (1) the $q_{H}-q_{L}=Q_{j}\left(q_{L}\right)$ functions in Figure 7 are well-defined, continuous and increasing; (2) $Q_{j}$ hits each boundary $A_{j} \cap A_{j+1}$ and $A_{j} \cap A_{j-1}$ exactly once; and (3) $Q_{j}$ hits $Q_{j+1}$ exactly once, and it is in $A_{j} \cap A_{j+1}$, except $Q_{3}$ and $Q_{4}$ which are connected by $Q_{34}$ along $A_{3} \cap A_{4}$.

Lemma 5 simply confirms that Figure 7 must look the way we drew it. Given this, we can establish:

Proposition 9 In the integrated model, generically in $\left(\delta_{L}, \delta_{H}\right)$ space, there is a unique equilibrium. If $\delta_{L}>\bar{\delta}$ the equilibrium is $\phi_{0}$. If $\delta_{L}<\bar{\delta}$ there exist functions $\delta_{01}\left(\delta_{L}\right) \geq \delta_{12}\left(\delta_{L}\right) \geq \delta_{23}\left(\delta_{L}\right) \geq \delta_{3 \Phi}\left(\delta_{L}\right) \geq \delta_{\Phi 4}\left(\delta_{L}\right) \geq \delta_{L}$, with equality iff $\delta_{L}=\bar{\delta}$, as shown in Figure 8, with the following properties:

1. $\delta_{H}>\delta_{01}\left(\delta_{L}\right) \Rightarrow \phi_{0}$ is the equilibrium

2. $\delta_{01}\left(\delta_{L}\right)>\delta_{H}>\delta_{12}\left(\delta_{L}\right) \Rightarrow \phi_{1}$ is the equilibrium

3. $\delta_{12}\left(\delta_{L}\right)>\delta_{H}>\delta_{23}\left(\delta_{L}\right) \Rightarrow \phi_{2}$ is the equilibrium

4. $\delta_{23}\left(\delta_{L}\right)>\delta_{H}>\delta_{3 \Phi}\left(\delta_{L}\right) \Rightarrow \phi_{3}$ is the equilibrium

5. $\delta_{3 \Phi}\left(\delta_{L}\right)>\delta_{H}>\delta_{\Phi 4}\left(\delta_{L}\right) \Rightarrow \phi_{34}$ is the equilibrium

6. $\delta_{\Phi 4}\left(\delta_{L}\right)>\delta_{H}>\delta_{L} \Rightarrow \phi_{4}$ is the equilibrium. 


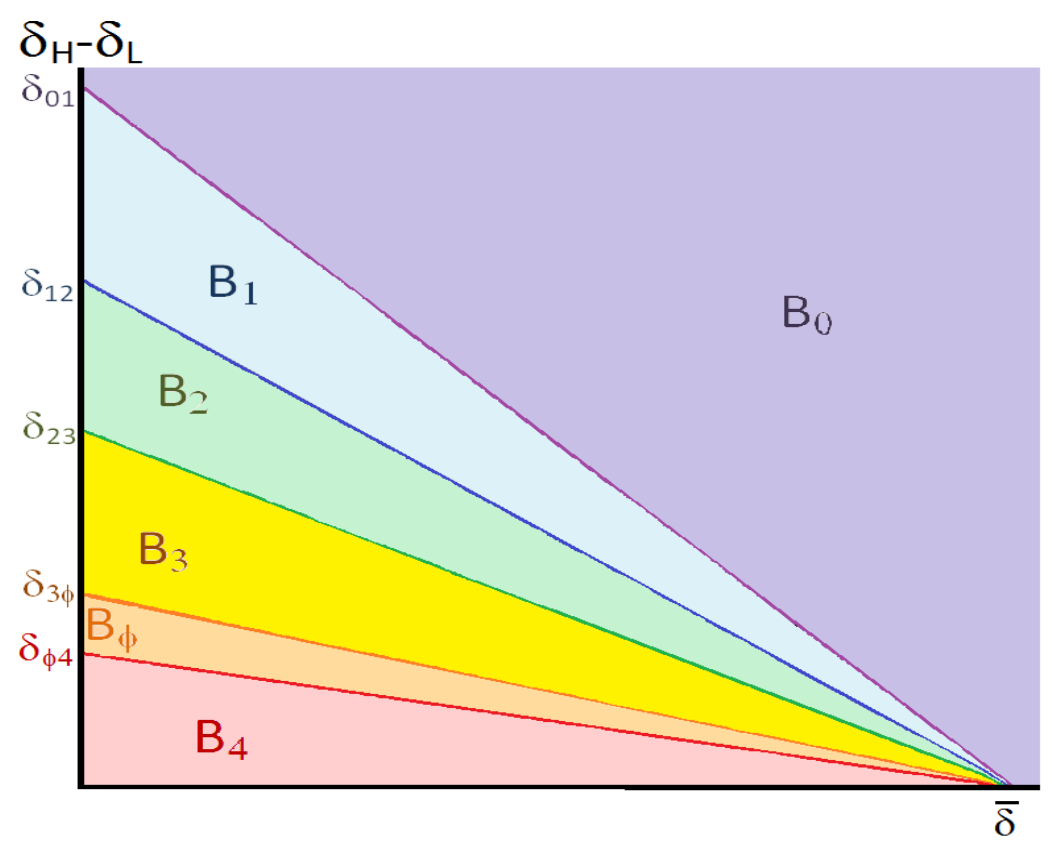

Figure 8: Equilibria in $\left(\delta_{L}, \delta_{H}-\delta_{L}\right)$ space.

Proof: As discussed above, $\delta_{L}>\bar{\delta}$ implies $\phi_{0}$ is the unique equilibrium. Consider a fixed $\delta_{L}<\bar{\delta}$. If $\delta_{H}$ is big $\phi_{0}$ is still the unique equilibrium, with $\left(q_{L}, q_{H}\right)$ given by (43). By reducing $\delta_{H}$ the $\phi_{0}$ equilibrium traces a path $Q_{0}$ through $A_{0}$ as in Figure

7. It is easy to check $Q_{0}$ is a straight line with slope greater than 1 . So at some $\delta_{H}=\delta_{01}\left(\delta_{L}\right), Q_{0}$ hits the boundary $A_{0} \cap A_{1}$ before it hits the horizontal axis. Hence, $\delta_{01}\left(\delta_{L}\right)>\delta_{L}$. On the boundary $A_{0} \cap A_{1}$, with $\delta_{H}=\delta_{01}\left(\delta_{L}\right)$, there are three equilibria: $\phi_{0}, \phi_{1}$ and $\phi_{01}$. Reducing $\delta_{H}$ further the $\phi_{1}$ equilibrium traces $Q_{1}$ through $A_{1}$ until it hits the boundary $A_{1} \cap A_{2}$ at some $\delta_{H}=\delta_{12}\left(\delta_{L}\right)$, where $\delta_{L}<\delta_{12}\left(\delta_{L}\right)<\delta_{01}\left(\delta_{L}\right)$. At $\delta_{H}=\delta_{12}\left(\delta_{L}\right)$ there are three equilibria: $\phi_{1}, \phi_{2}$ and $\phi_{12}$. Reducing $\delta_{H}$ further the $\phi_{2}$ equilibrium traces $Q_{2}$ through $A_{2}$ until it hits $A_{2} \cap A_{3}$ at $\delta_{H}=\delta_{23}\left(\delta_{L}\right)$, where $\delta_{L}<\delta_{23}\left(\delta_{L}\right)<\delta_{12}\left(\delta_{L}\right)$. At $\delta_{23}\left(\delta_{L}\right)$ there are three equilibria: $\phi_{2}, \phi_{3}$ and $\phi_{23}$.

Reducing $\delta_{H}$ further the $\phi_{3}$ equilibrium traces $Q_{3}$ through $A_{3}$ until it hits $A_{3} \cap A_{4}$ at $\delta_{H}=\delta_{3 \Phi}\left(\delta_{L}\right)$, where $\delta_{L}<\delta_{3 \Phi}\left(\delta_{L}\right)<\delta_{23}\left(\delta_{L}\right)$. For $\delta_{H}<\delta_{3 \Phi}\left(\delta_{L}\right)$, neither $\phi_{3}$ nor $\phi_{4}$ is an equilibrium, as discussed above, and agents must mix, $\phi_{H H} \in(0,1)$. As we reduce $\delta_{H}$ the probability $\phi_{H H}$ increases and the $\phi_{34}$ equilibrium traces $Q_{34}$ along 
$A_{3} \cap A_{4}$, until $\phi_{H H}=1$ and $Q_{34}$ meets $Q_{4}$ at $\delta_{H}=\delta_{\Phi 4}\left(\delta_{L}\right)$, where $\delta_{L}<\delta_{\Phi 4}\left(\delta_{L}\right)<$ $\delta_{3 \Phi}\left(\delta_{L}\right)$. Finally, reducing $\delta_{H}$ further, all the way to $\delta_{H}=\delta_{L}$, the $\phi_{4}$ equilibrium traces $Q_{4}$ through $A_{4}$. We know $\phi_{4}$ is the unique equilibrium when $\delta_{H}$ is close to $\delta_{L}$, since $\delta_{H}=\delta_{L}$ is STW, which has a unique equilibria given the parameter conditions assumed here, $\theta=1$ and $\delta_{L}>0$.

Summarizing, for any $\delta_{L}<\bar{\delta}$, there are cutoffs for $\delta_{H}$ partitioning parameter space into regions where the different equilibria exist, as shown in Figure 8.

Trade patterns here are more interesting than in the basic DGP and STW setups. Agents have to decide when to give up their assets to get $q$, depending on how much they like the asset, and on how much $q$ they get, which depends on how much a counterparty likes the asset. Nonlinear $u(q)$ is important for the results, but so is $\delta$ switching. Notice that since STW can have multiple steady states and interesting dynamics, this is also true for some parameters in the integrated model, simply by continuity. Proposition 9 does not admit these possibilities because of the maintained parameter restrictions, $\theta=1$ and $\delta_{H} \geq \delta_{L}>0$, which put us in the the left panel of Figure 4 in our analysis of STW. But we can relax these restrictions. If $\theta<1$ there are multiple steady states and interesting dynamics in STW when $\delta>\hat{\delta}$; and if $\theta=1$ there are similar outcomes when $\delta<0$. This observation establishes the following:

Proposition 10 For some parameters the integrated model admits multiple steady states, plus nontrivial deterministic and stochastic dynamic equilibria.

If people in finance are interested in multiplicity, bubbles, excess volatility and so on, they should find this interesting. The standard DGP model of OTC markets cannot generate such phenomena; a nonlinear version can. Characterizing the set of equilibria in the integrated model more generally - i.e., for all $\theta$ and $\delta$ - seems worth additional effort. Also, as mentioned, in Appendix A we allow $\delta \in\left\{\delta_{1}, \delta_{2}, \ldots \delta_{I}\right\}$ for any $I$, and provide an algorithm for finding the set of pure-strategy steady-state equilibria. It may be interesting to study dynamics in this case, too. In terms of 
monetary economics, once we allow time-varying $\delta$ 's, there emerges a role for various types of intermediation, as well as transitional dynamics, and both of these seem worth investigating. All of this must be left to future research.

\section{Summary and Conclusion}

We presented many Propositions, some similar to findings in existing papers, and some new. Reestablishing a few existing results was necessary before we could generalize and integrate the models. In STW, we derived several stronger or different results than previous analyses - e.g., we gave a complete characterization of steady states and perfect-foresight dynamics for all $\delta$, we provided an alternative method for verifying that sunspot equilibria exist, and we did the whole thing using a different, and for this application, arguably better, bargaining solution. In DGP, we reviewed standard results, and provided some new twists - e.g., we introduced intermediaries that can issue or hold assets, which lead to some insights about inside and outside liquidity. We also established the uniqueness of equilibria, not only steady states, in DGP. The Introduction lists other results that are new.

One contribution, we think, was simply to compare and contrast two workhorse models in monetary and financial economics. Users of STW or DGP - and there are many, as documented in fn. 1 - ought to know about this connection. Another contribution was to embed the models into a simple general equilibrium context, generating interactions between financial and other markets. For the integrated model nesting STW and DGP, we gave a characterization of the equilibrium set at least for steady states and a particular bargaining solution. More can be done. The goals of this paper were to develop a framework that allows one to think about extensions and applications, to understand search-and-bargaining theory at a deeper level, and to bridge some gaps between studies of money and finance. 


\section{Appendices}

A. The General Integrated Model: Let $\boldsymbol{\delta}=\left(\delta_{1}, \delta_{2}, \ldots \delta_{I}\right)$, for any integer $I$, with $\delta_{j}>\delta_{i}$ for $j>i$. Let $\boldsymbol{\Lambda}=\left[\lambda_{i j}\right]$ be a matrix of Poisson switching rates and $\boldsymbol{\Phi}=\left[\phi_{i j}\right]$ a matrix of trading strategies. Let $n_{m}^{i}$ be the measure of agents with $\delta=\delta_{i}$ and $m \in\{0,1\}$. The laws of motion for $n_{1}^{i}$ and $n_{0}^{i}$ are

$$
\begin{aligned}
& \dot{n}_{1}^{i}=\sum_{k} \lambda_{k i} n_{1}^{k}-n_{1}^{i} \sum_{k} \lambda_{i k}+\sigma n_{0}^{i} \sum_{k} n_{1}^{k} \phi_{k i}-\sigma n_{1}^{i} \sum_{k} n_{0}^{k} \phi_{i k} \\
& \dot{n}_{0}^{i}=\sum_{k} \lambda_{k i} n_{0}^{k}-n_{0}^{i} \sum_{k} \lambda_{i k}-\sigma n_{0}^{i} \sum_{k} n_{1}^{k} \phi_{k i}+\sigma n_{1}^{i} \sum_{k} n_{0}^{k} \phi_{i k}
\end{aligned}
$$

with identities $\sum_{i} n_{1}^{i}=M$ and $\sum_{i} n_{0}^{i}=1-M$. Bellman's equations are

$$
\begin{aligned}
& r V_{1}^{i}=\sigma \sum_{k} n_{0}^{k} \phi_{i k}\left[u\left(q_{i k}\right)-\Delta^{i}\right]+\sum_{k} \lambda_{i k}\left(V_{1}^{k}-V_{1}^{j}\right)+\delta_{i}+\dot{V}_{1}^{i} \\
& r V_{0}^{i}=\sigma \sum_{k} n_{1}^{k} \phi_{k i}\left[\Delta^{i}-c\left(q_{k i}\right)\right]+\sum_{k} \lambda_{i k}\left(V_{0}^{k}-V_{0}^{j}\right)+\dot{V}_{0}^{i}
\end{aligned}
$$

where in the baseline model $\Delta^{i}=V_{1}^{i}-V_{0}^{i}$, and in the GE extension $\Delta^{i}=W_{1}^{i}-W_{0}^{i}$. The bargaining solution is $g\left(q_{i j}\right)=\theta \Delta^{j}+(1-\theta) \Delta^{i}$. The trading rules are: $\phi_{i j}=1$ if $u\left(q_{i j}\right)>\Delta^{i}$ and $c\left(q_{i j}\right) \leq \Delta^{j}$, etc.

Subtracting the value functions,

$$
\begin{aligned}
r \Delta^{i}= & \sigma \sum_{k} n_{0}^{k} \phi_{i k}\left[u\left(q_{i k}\right)-\Delta^{i}\right]-\sigma \sum_{k} n_{1}^{k} \phi_{k i}\left[\Delta^{i}-c\left(q_{k i}\right)\right] \\
& +\sum_{k} \lambda_{i k}\left(\Delta^{k}-\Delta^{i}\right)+\delta_{i}+\dot{\Delta}^{i}
\end{aligned}
$$

Assume $u(q)=c(q)=q$, for now. Then eliminate $q_{i j}=\theta \Delta^{j}+(1-\theta) \Delta^{i}$ to get the simple expression

$$
r \Delta^{i}=\sigma \sum_{k}\left[\theta n_{0}^{k} \phi_{i k}+(1-\theta) n_{1}^{k} \phi_{k i}+\lambda_{i k}\right]\left(\Delta^{k}-\Delta^{i}\right)+\delta_{i}+\dot{\Delta}^{i},
$$

a linear differential equation in $\Delta=\left(\Delta^{1}, \Delta^{2}, \ldots \Delta^{N}\right)$. Also, it is clear that $\phi_{i j}=1 \Leftrightarrow$ $\Delta^{j}>\Delta^{i}$, ignoring borderline cases where $u\left(q^{i j}\right)=\Delta^{i}$ or $c\left(q^{i j}\right)=\Delta^{j}$ (or adjusting the condition to accommodate such cases).

Here is an algorithm for solving for the set of pure-strategy steady-state equilibria. First fill in $\mathbf{\Phi}$ with all 0's. Then solve (44)-(45) for steady state $\mathbf{n}=\left(\mathbf{n}_{0}, \mathbf{n}_{1}\right)$. Insert 
$\boldsymbol{\Phi}$ and $\mathbf{n}$ into (49) with $\dot{\Delta}=\mathbf{0}$ and solve for $\boldsymbol{\Delta}$. Check if the solution satisfies $\phi_{i j}=1 \Leftrightarrow \Delta^{j}>\Delta^{i}$ for all $i, j$ given $\boldsymbol{\Phi}$. If it does, $\boldsymbol{\Phi}$ is an equilibrium; otherwise, it is not. Then update by changing one $\phi_{i j}$ from 0 to 1 , and repeat until every $\phi_{i j}$ is 1 .

With nonlinear utility, the only difference is that one has to solve the nonlinear system (48) together with the bargaining solution $g\left(q_{i j}\right)=\theta \Delta^{j}+(1-\theta) \Delta^{i}$ simultaneously for $\boldsymbol{\Delta}$ and $\mathbf{q}=\left[q_{i j}\right]$, and replace $\phi_{i j}=1 \Leftrightarrow \Delta^{j}>\Delta^{i}$ with $\phi_{i j}=1 \Leftrightarrow u\left(q_{i j}\right)>\Delta^{i}$ and $c\left(q_{i j}\right)<\Delta^{j}$, if we ignore (or adjust for) borderline cases where $u\left(q_{i j}\right)=\Delta^{i}$ or $c\left(q_{i j}\right)=\Delta^{j}$.

B. Proof of Proposition 2: Subtracting the Bellman equations and using the bargaining solution $g\left(q_{i}\right)=V_{1}^{i}-V_{0}^{i}$, we get

$$
r g\left(q_{i}\right)=\sigma(1-M) u\left(q_{i}\right)+\sigma M c\left(q_{i}\right)+\delta-\sigma g\left(q_{i}\right)+\varepsilon_{i}\left[g\left(q_{j}\right)-g\left(q_{i}\right)\right],
$$

for $i=1,2$. It is clear that we can always ignore $s$, and set $q_{1}=q_{2}=q$, in which case (50) becomes $e(q)=0$ from the perfect foresight model. A proper sunspot equilibrium is a pair $\left(q_{1}, q_{2}\right)$, with $q_{1} \neq q_{2}$, solving (50) for $i=1,2$. While is natural to interpret $\left(q_{1}, q_{2}\right)$ as endogenously determined functions of $\left(\varepsilon_{1}, \varepsilon_{2}\right)$, we we instead solve $(50)$ for $\left(\varepsilon_{1}, \varepsilon_{2}\right)$ as functions of $\left(q_{1}, q_{2}\right)$ :

$$
\varepsilon_{1}=\frac{e\left(q_{1}\right)}{g\left(q_{2}\right)-g\left(q_{1}\right)} \text { and } \varepsilon_{2}=\frac{-e\left(q_{2}\right)}{g\left(q_{2}\right)-g\left(q_{1}\right)}
$$

For any $q_{1} \in(0, \bar{q})$ and $q_{2} \in\left(q_{1}, \bar{q}\right)$, there is a stationary sunspot equilibrium with these values of $\left(q_{1}, q_{2}\right)$, and $\left(\varepsilon_{1}, \varepsilon_{2}\right)$ solving (51), as long as $\varepsilon_{1}, \varepsilon_{1}>0$. This requires that $e(q)$ is decreasing around a solution to $e(q)=0$. There are exactly two scenarios in which this obtains:

Case 1. $\theta \in\left(\theta_{u}, 1\right]$ and $\delta \in(\underline{\delta}, 0)$, as in the fourth panel of Figure 1 . In this situation, for any $q_{1} \in\left(0, q_{L}^{e}\right)$ and $q_{2} \in\left(q_{L}^{e}, q_{H}^{e}\right)$, there is an equilibrium where $q_{t}$ fluctuates around the low-level steady state $q_{L}^{e}$ with arrival rates given by (51). This is so because, as is clear from the Figure, $q_{2}>q_{1}$ and $e\left(q_{2}\right)<e\left(q_{1}\right)$, which guarantees $\varepsilon_{1}, \varepsilon_{2}>0$. 
Case 2. $\theta \in\left[0, \theta_{c}\right)$ and $\delta \in(\bar{\delta}, \hat{\delta})$, as in the second panel of Figure 3. Now for any $q_{1} \in\left(q_{L}^{e}, q_{H}^{e}\right)$ and $q_{2} \in\left(q_{H}^{e}, \bar{q}\right)$, there is an equilibrium where $q_{t}$ fluctuates around the high-level steady state $q_{H}^{e}$ with arrival rates given by (51), for reasons similar to the previous case.

C. Proof of Lemma 3: Using $\gamma_{H}=\lambda_{L} /\left(\lambda_{L}+\lambda_{H}\right)$, (21)-(22) can be written:

$$
\begin{aligned}
& n_{0}^{H}=\frac{1}{2}\left[\frac{\lambda_{L}}{\lambda_{L}+\lambda_{H}}-M-\lambda_{L}-\lambda_{H}+\sqrt{\left(\frac{\lambda_{L}}{\lambda_{L}+\lambda_{H}}-M+\lambda_{L}+\lambda_{H}\right)^{2}+4 \lambda_{H} M}\right] \\
& n_{1}^{L}=\frac{1}{2}\left[-\frac{\lambda_{L}}{\lambda_{L}+\lambda_{H}}+M-\lambda_{L}-\lambda_{H}+\sqrt{\left(\frac{\lambda_{L}}{\lambda_{L}+\lambda_{H}}-M+\lambda_{L}+\lambda_{H}\right)^{2}+4 \lambda_{H} M}\right]
\end{aligned}
$$

Letting $D=\left(\gamma_{H}-M+\lambda_{L}+\lambda_{H}\right)^{2}+4 \lambda_{H} M$, we have

$$
\begin{aligned}
\frac{\partial n_{0}^{H}}{\partial \lambda_{H}} & =-\frac{\sqrt{D}+\gamma_{H}-M-\lambda_{L}-\lambda_{H}}{2 \sqrt{D}}\left[\frac{\lambda_{L}}{\left(\lambda_{L}+\lambda_{H}\right)^{2}}+1\right]<0 \\
\frac{\partial n_{1}^{L}}{\partial \lambda_{L}} & =\frac{\gamma_{H}-M+\lambda_{L}+\lambda_{H}-\sqrt{D}}{2 \sqrt{D}}\left[1+\frac{\lambda_{H}}{\left(\lambda_{L}+\lambda_{H}\right)^{2}}\right]>0 .
\end{aligned}
$$

For the next result,

$$
\frac{\partial n_{0}^{H}}{\partial \lambda_{L}}=\frac{\sqrt{D}+\gamma_{H}-M-\lambda_{L}-\lambda_{H}}{2 \sqrt{D}}\left[\frac{\lambda_{H}}{\left(\lambda_{L}+\lambda_{H}\right)^{2}}-1\right]+\frac{1-M}{\sqrt{D}} .
$$

Notice $\lambda_{H} /\left(\lambda_{L}+\lambda_{H}\right)^{2}-1=\left(1-\gamma_{H}-\lambda_{L}-\lambda_{H}\right) /\left(\lambda_{L}+\lambda_{H}\right)$. If $1-\gamma_{H}-\lambda_{L}-\lambda_{H}>0$ then $\partial n_{0}^{H} / \partial \lambda_{L}>0$. So suppose $1-\gamma_{H}-\lambda_{L}-\lambda_{H}<0$. Then $\partial n_{0}^{H} / \partial \lambda_{L}>0$ iff

$$
\left(1-\gamma_{H}-\lambda_{L}-\lambda_{H}\right)\left(\sqrt{D}+\gamma_{H}-M-\lambda_{L}-\lambda_{H}\right)+2(1-M)\left(\lambda_{L}+\lambda_{H}\right)>0
$$

After some algebra, this holds iff

$$
\left(1-\gamma_{H}-\lambda_{L}-\lambda_{H}\right)^{2}+(1-M)>2\left(1-\gamma_{H}-\lambda_{L}-\lambda_{H}\right)
$$

which is true under the supposition $1-\gamma_{H}-\lambda_{L}-\lambda_{H}<0$. So $\partial n_{0}^{H} / \partial \lambda_{L}>0$.

Finally,

$$
\frac{\partial n_{1}^{L}}{\partial \lambda_{H}}=\frac{\sqrt{D}+\gamma_{H}-M+\lambda_{L}+\lambda_{H}}{2 \sqrt{D}}\left[\frac{\lambda_{L}}{\left(\lambda_{H}+\lambda_{L}\right)^{2}}-1\right]+\frac{M}{\sqrt{D}} .
$$


Again, if $\gamma_{H}-\lambda_{L}-\lambda_{H}>0$ then $\partial n_{1}^{L} / \partial \lambda_{L}>0$. So suppose $\gamma_{H}-\lambda_{L}-\lambda_{H}<0$. Then

$$
\frac{\partial n_{1}^{L}}{\partial \lambda_{H}} \approx\left(\gamma_{H}-\lambda_{L}-\lambda_{H}\right)^{2}+M-2\left(\gamma_{H}-\lambda_{L}-\lambda_{H}\right)>0
$$

under the supposition $1-\gamma_{H}-\lambda_{L}-\lambda_{H}<0$. This establishes the claim.

D. Steady States with Brokers and Bankers: We provide the solutions to the steady-state equations for DGP with intermediation. With brokers,

$$
\begin{aligned}
n_{0}^{H} & =\frac{1}{2}\left[\gamma_{H}-M-\lambda_{H}-\lambda_{L}-\beta+\sqrt{\bar{D}}\right] \\
n_{1}^{L} & =\frac{1}{2}\left[-\gamma_{H}+M-\lambda_{H}-\lambda_{L}-\beta+\sqrt{\bar{D}}\right] \\
n_{1}^{H} & =\frac{1}{2}\left[\gamma_{H}+M+\lambda_{H}+\lambda_{L}+\beta-\sqrt{\bar{D}}\right] \\
n_{0}^{L} & =\frac{1}{2}\left[2-\gamma_{H}-M+\lambda_{H}+\lambda_{L}+\beta-\sqrt{\bar{D}}\right]
\end{aligned}
$$

where $\bar{D}=\left(\gamma_{H}-M+\lambda_{H}+\lambda_{L}+\beta\right)^{2}+4\left[\lambda_{H} M-\beta\left(\gamma_{H}-M\right)\right]$. With bankers,

$$
\begin{aligned}
n_{0}^{H} & =n_{1}^{L}=\frac{1}{2}\left[-\left(\lambda_{H}+\lambda_{L}+\beta\right)+\sqrt{\tilde{D}}\right] \\
n_{1}^{H} & =\frac{1}{2}\left[2 \gamma_{H}+\lambda_{H}+\lambda_{L}+\beta-\sqrt{\tilde{D}}\right] \\
n_{0}^{L} & =\frac{1}{2}\left[2\left(1-\gamma_{H}\right)+\lambda_{H}+\lambda_{L}+\beta-\sqrt{\tilde{D}}\right]
\end{aligned}
$$

and $N=M-\gamma_{H}$, where $\tilde{D}=\left(\beta+\lambda_{H}+\lambda_{L}\right)^{2}+4 \lambda_{H} \gamma_{H}$.

E. Nonseparable Utility: We derive the effects of $\delta$ on equilibrium in the GE version of STW with nonseparable preferences, $u(q, x)$ and $v(q, x)$. Agents in the Walrasian market solve

$$
W_{m}=\max \left\{-\ell+(1+r)^{-1} V_{m^{\prime}}(x)\right\} \text { st } x=\ell+(\delta+p) m-p m^{\prime} \text { and } m^{\prime} \in\{0,1\} \text {, }
$$

which implies $W_{1}-W_{0}=p+\delta$. The OTC Bellman equations are

$$
\begin{aligned}
& V_{1}\left(x_{1}\right)=\sigma(1-M)\left(u^{T}+W_{0}\right)+[1-\sigma(1-M)]\left(u^{N}+W_{1}\right) \\
& V_{0}\left(x_{0}\right)=\sigma M\left(v^{T}+W_{1}\right)+(1-\sigma M)\left(v^{N}+W_{0}\right),
\end{aligned}
$$


where superscripts $T$ and $N$ indicate traders and nontraders: $u^{T}=u\left(q, x_{1}\right), u^{N}=$ $u\left(0, x_{1}\right)$, etc. Also, agents either choose $m^{\prime}=1$ and $x=x_{1}$ or $m^{\prime}=0$ and $x=x_{0}$. The FOC for $x_{1}$ and $x_{0}$ are

$$
\begin{aligned}
& (1+r)=V_{1}^{\prime}\left(x_{1}\right)=\sigma(1-M) u_{x}^{T}+[1-\sigma(1-M)] u_{x}^{N} \\
& (1+r)=V_{0}^{\prime}\left(x_{0}\right)=\sigma M v_{x}^{T}+(1-\sigma M) v_{x}^{N} .
\end{aligned}
$$

Indifference between $m^{\prime}=1$ and $m^{\prime}=0$ implies $-p-x_{1}+(1+r)^{-1} V_{1}\left(x_{1}\right)=-x_{0}+$ $(1+r)^{-1} V_{0}\left(x_{0}\right)$, or:

$p=\frac{\sigma(1-M)\left(u^{T}-u^{N}\right)-\sigma M\left(v^{T}-v^{N}\right)+u^{N}-v^{N}+(1+r)\left(x_{0}-x_{1}\right)+(1-\sigma) \delta}{r+\sigma}$

OTC bargaining implies $(1-\theta)\left(u^{T}-u^{N}\right)-\theta\left(v^{T}-v^{N}\right)=W_{1}-W_{0}=p+\delta$. Eliminating $p$ using the previous condition, we get a generalization of (40)

$$
A_{u}\left(u^{T}-u^{N}\right)+A_{c}\left(v^{T}-v^{N}\right)+v^{N}-u^{N}+(1+r)\left(x_{1}-x_{0}-\delta\right)=0 .
$$

Steady state $\left(q, x_{1}, x_{0}\right)$ solves (52)-(54). From this we derive $\boldsymbol{\Gamma}\left(d q, d x_{1}, d x_{0}\right)^{\prime}=$ $(d \tilde{\delta}, 0,0)^{\prime}$, where

$$
\boldsymbol{\Gamma}=\left[\begin{array}{ccc}
A_{u} u_{q}^{T}+A_{c} v_{q}^{T} & A_{u} u_{x}^{T}-\left(1+A_{u}\right) u_{x}^{N}+(1+r) & A_{c} v_{x}^{T}+\left(1-A_{c}\right) v_{x}^{N}-(1+r) \\
\sigma(1-M) u_{x q}^{T} & E u_{x x} & 0 \\
\sigma M v_{x q}^{T} & 0 & E v_{x x}
\end{array}\right]
$$

with $E u_{x x}=\sigma(1-M) u_{x x}^{T}+[1-\sigma(1-M)] u_{x x}^{N}$ and $E v_{x x}=\sigma M v_{x x}^{T}+(1-\sigma M) v_{x x}^{N}$. Letting $\Omega=\operatorname{det}(\boldsymbol{\Gamma})$, we have

$$
\frac{\partial q}{\partial \tilde{\delta}}=\frac{E u_{x x} E v_{x x}}{\Omega}, \frac{\partial x_{1}}{\partial \tilde{\delta}}=\frac{-E v_{x x} \sigma(1-M) u_{x q}^{T}}{\Omega} \text { and } \frac{\partial x_{0}}{\partial \tilde{\delta}}=\frac{-E u_{x x} \sigma M v_{x q}^{T}}{\Omega} .
$$

In general, $\Omega$ is complicated, but if $u_{q x}=v_{q x}=0$ then

$$
\Omega=E u_{x x} E v_{x x}\left(A_{u} u_{q}^{T}+A_{c} v_{q}^{T}\right) .
$$

If there is a unique steady state then $\Omega \approx A_{u} u_{q}^{T}+A_{c} v_{q}^{T}=\tilde{e}^{\prime}(q)>0$. Given this, we assume $\Omega>0$ even in the nonseparable case (which is reasonable, since our main 
intention is to show what might happen with nonseparable utility). Then $\partial q / \partial \tilde{\delta}>0$, $\partial x_{1} / \partial \tilde{\delta} \approx u_{x q}^{T}$ and $\partial x_{0} / \partial \tilde{\delta} \approx v_{x q}^{T}$, as reported in the text.

F. Proof of Lemma 2: The Jacobian matrix for (32)-(35) is

$$
\mathbf{J}=\left[\begin{array}{cccc}
J_{11} & -\lambda_{H}-(1-\theta) n_{1}^{L} & 0 & (1-\theta)\left(\Delta_{H}-\Delta_{L}\right) \\
-\lambda_{L}-n_{0}^{H} \theta & J_{22} & -\theta\left(\Delta_{H}-\Delta_{L}\right) & 0 \\
0 & 0 & -n_{1}^{L}-\lambda_{L}-\lambda_{H} & -n_{0}^{H} \\
0 & 0 & -n_{1}^{L} & -n_{0}^{H}-\lambda_{L}-\lambda_{H}
\end{array}\right]
$$

where $J_{11}=r+\lambda_{H}+(1-\theta) n_{1}^{L}$ and $J_{22}=r+\lambda_{L}+n_{0}^{H} \theta$. We seek the roots of $\left|\mathbf{J}_{\mu}\right|=0$, where

$$
\begin{aligned}
& \left|\mathbf{J}_{\mu}\right|=\left|\begin{array}{cccc}
J_{11}-\mu & -\lambda_{H}-(1-\theta) n_{1}^{L} & 0 & (1-\theta)\left(\Delta_{H}-\Delta_{L}\right) \\
-\lambda_{L}-n_{0}^{H} \theta & J_{22}-\mu & -\theta\left(\Delta_{H}-\Delta_{L}\right) & 0 \\
0 & 0 & -n_{1}^{L}-\lambda_{L}-\lambda_{H}-\mu & -n_{0}^{H} \\
0 & 0 & -n_{1}^{L} & -n_{0}^{H}-\lambda_{L}-\lambda_{H}-\mu
\end{array}\right| \\
& =n_{1}^{L}\left|\begin{array}{ccc}
J_{11}-\mu & -\lambda_{H}-(1-\theta) n_{1}^{L} & (1-\theta)\left(\Delta_{H}-\Delta_{L}\right) \\
-\lambda_{L}-n_{0}^{H} \theta & J_{22}-\mu & 0 \\
0 & 0 & -n_{0}^{H}
\end{array}\right| \\
& -\left(n_{0}^{H}+\lambda_{L}+\lambda_{H}+\mu\right)\left|\begin{array}{ccc}
J_{11}-\mu & -\lambda_{H}-(1-\theta) n_{1}^{L} & 0 \\
-\lambda_{L}-n_{0}^{H} \theta & J_{22}-\mu & -\theta\left(\Delta_{H}-\Delta_{L}\right) \\
0 & 0 & -n_{1}^{L}-\lambda_{L}-\lambda_{H}-\mu
\end{array}\right| \\
& =-n_{1}^{L} n_{0}^{H}\left|\begin{array}{cc}
J_{11}-\mu & -\lambda_{H}-(1-\theta) n_{1}^{L} \\
-\lambda_{L}-n_{0}^{H} \theta & J_{22}-\mu
\end{array}\right| \\
& +\left(n_{0}^{H}+\lambda_{L}+\lambda_{H}+\mu\right)\left(n_{1}^{L}+\lambda_{L}+\lambda_{H}+\mu\right)\left|\begin{array}{cc}
J_{11}-\mu & -\lambda_{H}-(1-\theta) n_{1}^{L} \\
-\lambda_{L}-n_{0}^{H} \theta & J_{22}-\mu
\end{array}\right| \\
& =\left(\lambda_{L}+\lambda_{H}+\mu\right)\left(n_{0}^{H}+n_{1}^{L}+\lambda_{L}+\lambda_{H}+\mu\right)\left|\begin{array}{cc}
J_{11}-\mu & -\lambda_{H}-(1-\theta) n_{1}^{L} \\
-\lambda_{L}-n_{0}^{H} \theta & J_{22}-\mu
\end{array}\right|
\end{aligned}
$$

It is clear there are two negative eigenvalues, the values of $\mu$ that zero the first two factors: $\mu_{1}=-\left(\lambda_{L}+\lambda_{H}\right)<0$ and $\mu_{2}=-\left(n_{0}^{H}+n_{1}^{L}+\lambda_{L}+\lambda_{H}\right)<0$. The other two eigenvalues are the roots of the determinant of the $2 \times 2$ matrix:

$$
\begin{aligned}
0 & =\left(r+\lambda_{L}+n_{0}^{H} \theta-\mu\right)\left[r+\lambda_{H}+(1-\theta) n_{1}^{L}-\mu\right]-\left(\lambda_{L}+n_{0}^{H} \theta\right)\left[\lambda_{H}+(1-\theta) n_{1}^{L}\right] \\
& =(r-\mu)^{2}+(r-\mu)\left[\lambda_{L}+n_{0}^{H} \theta+\lambda_{H}+(1-\theta) n_{1}^{L}\right] \\
& =(r-\mu)\left[\lambda_{L}+n_{0}^{H} \theta+\lambda_{H}+(1-\theta) n_{1}^{L}+r-\mu\right] .
\end{aligned}
$$

Hence, the other two eigenvalues are positive: $\mu_{3}=r>0$ and $\mu_{4}=\lambda_{L}+n_{0}^{H} \theta+\lambda_{H}+$ $(1-\theta) n_{1}^{L}+r>0$. 


\section{References}

G. Afonso and R. Lagos (2012) "Trade Dynamics in the Market for Federal Funds," FRB NY Staff Report 594.

S.R. Aiyagari and N. Wallace (1991) "Existence of Steady States with Positive Consumption in the Kiyotaki-Wright Model," RES 58, 901-16.

L. Ales, F. Carapella, P. Maziero and W. Weber (2010) "A Model of Banknote Discounts," JET 142, 5-27.

S.B. Aruoba, G. Rocheteau and C. Waller (2007) "Bargaining and the Value of Money," JME 54, 2636-55.

A. Babus and P. Kondor (2012) "Trading and Information Diffusion in Over-theCounter Markets," mimeo.

Z. Bethune, P. Rupert and G. Rocheteau (2013) "Unemployment and Household Unsecured Debt," mimeo.

A. Berentsen (2002) "On the Distribution of Money Holdings in a Random-Matching Model," IER 43, 945-54.

A. Berentsen, G. Camera and C. Waller (2005) "The Distribution of Money Balances and the Nonneutrality of Money," IER 46, 465-487.

A. Berentsen, G. Menzio R. Wright (2011) "Inflation and Unemployment in the Long Run," AER 101, 371-398.

A. Berentsen, M. Molico and R. Wright (2002) "Indivisibilities, Lotteries and Monetary Exchange," JET 107, 70-94.

K. Burdett, A. Trejos and R. Wright (2001) "Cigarette Money," JET 99, 117-42.

R. Cavalcanti and N. Wallace (1999a) "A Model of Private Banknote Issue," RED 2, 104-136.

R. Cavalcanti and N. Wallace (1999b) "Inside and Outside Money as Alternative Media of Exchange," JMCB 31, 443-457.

J. Chiu and T. Koeppl (2012) "Trading Dynamics with Adverse Selection and Search," mimeo.

M. Choi (2012) "Asset Markets with Adverse Selection and Information Loss," mimeo.

M. Coles (1999) "Turnover Externalities with Marketplace Trading," IER 40, 851868. 
M. Coles and R. Wright (1998) "A Dynamic Model of Search, Bargaining, and Money," JET 78, 32-54.

E. Curtis and R. Wright (2004) "Price Setting, Price Dispersion, and the Value of Money; or, The Law of Two Prices," JME 51, 1599-621.

B. Craig and G. Rocheteau (2008) "State-Dependent Pricing, Inflation, and Welfare," European Econ Rev 52, 441-468.

D. Corbae, T. Temzelides and R. Wright (2003) "Directed Matching and Monetary Exchange," Econometrica 71, 731-56.

D. Duffie, N. Gârleanu and L. Pederson (2005) "Over-the-Counter Markets," Econometrica $73,1815-47$.

D. Duffie, N. Gârleanu and L. Pederson (2007) "Valuation in Over-the-Counter Markets," Rev. Financial Studies 20, 1865-1900.

H. Ennis (2001) "On Random Matching, Monetary Equilibria, and Sunspots," Macro. Dynamics 5, 132-42.

H. Ennis (2004) "Macroeconomic Fluctuations and Bargaining," JET 115, 322-40.

E. Green and R. Zhou (1998) "A Rudimentary Random-Matching Model with Divisible Money and Prices," JET 81, 252-71.

P. He, L. Huang and R. Wright (2005) "Money and Banking in Search Equilibrium," IER 46, 637-70.

B. Holmstrom and J. Tirole (2011) Inside and Outside Liquidity, MIT Press.

A. Hosios (1990) "On the Efficiency of Matching and Related Models of Search and Unemployment," RES 57, 279-298.

B. Julien, J. Kennes and I. King (2008) "Bidding For Money," JET 142, 196-217.

E. Kalai (1977) "Proportional Solutions to Bargaining Situations: Interpersonal Utility Comparisons," Econometrica 45, 1623-30.

N. Kiyotaki and J. Moore (1997) "Credit Cycles," JPE 105, 211-248.

N. Kiyotaki and R. Wright (1989) "On Money as a Medium of Exchange," JPE 97, 927-54.

N. Kocherlakota (1998) "Money is Memory," JET 81, 232-51.

R. Lagos and G. Rocheteau (2009) "Liquidity in Asset Markets with Search Frictions," Econometrica 77, 403-26 
R. Lagos, G. Rocheteau and P. Weill (2011) "Crises and Liquidity in Over-theCounter Markets," JET in press.

R. Lagos and R. Wright (2005) "A Unified Framework for Monetary Theory and Policy Analysis," JPE 113, 463-84.

B. Lester, A. Postlewaite and R. Wright (2012) "Liquidity, Information, Asset Prices and Monetary Policy," RES 79, 1209-38.

Y. Li (1998) "Middlemen and Private Information," JME 42, 131-159.

Y. Li (1999) "Money and Middlemen in an Economy with Private Information," Econ. Inquiry 37, 1-12.

R. Lucas (1978) "Asset Prices in an Exchange Economy," Econometrica 46, 1426-45.

A. Matsui and T. Shimizu (2005) "A Theory of Money with Market Places," IER $46,35-59$.

M. Molico (2006) "The Distribution of Money and Prices in Search Equilibrium," IER 47, 701-22.

C. Monnet and B. Narajabad (2012) "Why Rent When You Can Buy? A Theory of Repurchase Agreements," mimeo.

D. Mortensen and C. Pissarides (1994) "Job Creation and Job Destruction in the Theory of Unemployment," RES 61, 397-416.

E. Nosal and G. Rocheteau (2011) Money, Payments and Liquidity, MIT Press.

E. Nosal and N. Wallace (2007) "A Model of (the Threat of) Counterfeiting," JME 54, 994-1001.

E. Pagnotta and T. Philippon (2011) "Competing on Speed," mimeo.

N. Petrosky-Nadeau and G. Rocheteau (2013) "Unemployment, Financial Frictions, and the Housing Market," mimeo.

G. Rocheteau and T. Wong (2013) "A Continuous-Time Model of Monetary Exchange with Lumpy Consumption," mimeo.

G. Rocheteau and R. Wright (2005) "Money in Search Equilibrium, in Competitive Equilibrium, and in Competitive Search Equilibrium," Econometrica 73, 175202.

A. Rubinstein and A. Wolinsky (1987) "Middlemen," QJE 102, 581-94.

P. Rupert, M. Schindler and R. Wright (2001) "A Generalized Search-Theoretic Model of Monetary Exchange," JME 48, 605-22. 
A. Rodriguez-Lopez and G. Rocheteau (2013) "Liquidity Provision, Interest Rates, and Unemployment," mimeo.

A. Schevchenko (2004) "Middlemen," IER 45, 1-24.

S. Shi (1995) "Money and Prices: A Model of Search and Bargaining," JET 67, 467-96.

S. Shi (1997) “A Divisible Model of Fiat Money," Econometrica 65, 75-102.

J. Stiglitz (1990) "Symposium on Bubbles," JEP 4, 13-8.

A. Trejos and R. Wright (1993) "Search, Bargaining, Money and Prices: Recent Results and Policy Implications" JMCB 25, 558-76.

A. Trejos and R. Wright (1995) "Search, Bargaining, Money, and Prices," JPE 103, $118-41$.

A. Trejos and R. Wright (2001) "International Currency," BEJ Advances in Macro 1.

P. Weill and D. Vayanos (2008) "A Search-based Theory of the On-the-run Phenomenon," J. Finance 63, 1351-89.

F. Velde, W. Weber and R. Wright (1999) "A Model of Commodity Money, with Applications to Gresham's Law and the Debasement Puzzle," RED 2, 291-323.

N. Wallace (1980) "The Overlapping Generations Model of Fiat Money," in Models of Monetary Economies, ed. J. Kareken and N. Wallace.

N. Wallace (2000) "A Model of the Liquidity Yield Structure Based on Asset Indivisibility," JME 45, 55-68

N. Wallace (2010) "The Mechanism-design Approach to Monetary Economics," Handbook of Monetary Econ., ed. B. Friedman and M. Woodford.

N. Wallace and T. Zhu (2007a) "Pairwise Trade and Coexistence of Money and Higher-Return Assets," JET 133, 524-35.

N. Wallace and T. Zhu (2007b) "Float on a Note," JME 54, 229-46.

P. Weill (2007) "Leaning against the Wind," RES 74, 1329-54.

P. Weill (2008) "Liquidity Premia in Dynamic Bargaining Markets," JET 140, 66-96.

S. Williamson and R. Wright (2010) "New Monetarist Economics: Models," Handbook of Monetary Econ., ed. B. Friedman and M. Woodford. 